\title{
Efficient conjugate addition of carbonyl compounds to 3-nitro-2H-chromenes in the presence of bases
}

\author{
Zhi-peng Hu, Jun-min Zhang, Chun-liang Lou, Jin-jia Wang, Shao-zhen Nie, \\ and Ming Yan* \\ Institute of Drug Synthesis and Pharmaceutical Process, School of Pharmaceutical Sciences, \\ Sun Yat-sen University, Guangzhou 510006, China \\ E-mail: yanming@mail.sysu.edu.cn
}

DOI: http://dx.doi.org/10.3998/ark.5550190.0011.a03

\begin{abstract}
The conjugate addition of aldehydes and ketones to 3-nitro- $2 H$-chromenes under basic conditions has been explored. A series of inorganic and organic bases were examined. Proline combined with $\mathrm{NaOAc}$ was found to act as an efficient catalyst for the reaction. A number of substituted 3-nitro- $2 \mathrm{H}$-chromenes, aldehydes, and ketones are applicable for this transformation. trans-3-Nitro-4-substituted chromanes were sometimes obtained in good yields and with excellent diastereoselectivities. trans-2-Methyl-2-(3-nitrochroman-4-yl)propanal could be transformed into cis-2,3-dihydro-1,1-dimethyl-1H-chroman[3,4-b]pyrrole in one step.
\end{abstract}

Keywords: Conjugate addition, carbonyl compounds, 3-nitro-2H-chromenes, organocatalysis, proline

\section{Introduction}

Chromans are important structural units of many natural products and drugs. ${ }^{1}$ Chroman derivatives exhibit a variety of useful biological activities, ${ }^{2}$ such as antioxidation, ${ }^{3}$ antiestrogen, ${ }^{4}$ anticonvulsion, ${ }^{5}$ and neuroprotection. ${ }^{6}$ Their synthetic methods have been the subject of a number of publications. ${ }^{7} 3$-Nitro- $2 H$-chromenes, readily available from the reaction of salicylaldehyde and nitroethylene, are valuable intermediates for the synthesis of chroman derivatives. ${ }^{8}$ Their conjugate addition reactions with nucleophiles, ${ }^{9}$ reduction, ${ }^{1 \mathrm{a}}$ and 1,3 -dipolar cycloaddition with azomethine, azide, and diazo compounds, ${ }^{10}$ have been developed for the synthesis of many chroman derivatives. We speculate that the conjugate addition of unmodified carbonyl compounds to 3 -nitro- $2 H$-chromenes can provide a simple and direct route for chroman derivatives. To the best of our knowledge, such a transformation has never been explored. In this paper, we report highly efficient conjugate addition of aldehydes and ketones to 3-nitro- $2 \mathrm{H}$ - 
chromenes in the presence of bases. Proline combined with NaOAc was found to be a good catalyst for this reaction. A series of 3-nitrochromans was prepared, sometimes in good yields and with excellent diastereoselectivities.

\section{Results and Discussion}

Initially the reaction of 3-nitro- $2 \mathrm{H}$-chromene $\mathbf{1 a}$ and isobutyraldehyde $\mathbf{2 a}$ was studied. A number of inorganic and organic bases were examined as the catalyst. The results are summarized in Table 1. NaOAc and $\mathrm{K}_{2} \mathrm{CO}_{3}$ were found to be inefficient and no reaction was observed (Table 1, entries 1-2). Strong inorganic bases, such as $\mathrm{LiOH}$ and $\mathrm{KOH}$ provided low yield of product 3a together with many side products (Table 1, entries 3-4). Organic bases, such as triethylamine (TEA), $N, N$-diisopropylethylamine (DIPEA), 1,4-diazabicyclo[2.2.2]octane (DABCO), 1,8diazabicyclo[5.4.0] undec-7-ene (DBU), 4-dimethylaminopyridine (DMAP), and $\mathrm{N}$ methylpyrrolidine (NMP) gave 3a in poor yields (Table 1, entries 5-10). On the other hand, piperidine provided product 3a in significantly improved yield (Table 1, entry 11). Further study indicated that pyrrolidine is even a better base for the reaction (Table 1, entry 12). The results strongly imply that secondary amines exhibit beneficial effect for the transformation. Special base catalytic mechanism may not be involved in this case and the enamine activation mechanism is highly possible. In recent years, proline and its derivatives have been found to be efficient catalysts for many reactions of aldehydes and ketones via the enamine activation mechanism. ${ }^{11}$ Thus, proline was examined as the catalyst for the reaction of $\mathbf{1 a}$ and $\mathbf{2 a}$. Unfortunately, the reaction only provided 3a in low yield (Table 1, entry 13). Further study uncovered that the combination of $20 \mathrm{~mol} \%$ proline and $100 \mathrm{~mol} \%$ NaOAc produces an extremely efficient catalyst system. Product 3a was obtained in excellent yield (98\%) (Table 1, entry 14). In addition the reaction occurred with excellent diastereoselectivity and only trans-3a was observed. The trans-configuration of $\mathbf{3 a}$ was assigned by ${ }^{3} J_{(3-\mathrm{H}, 4-\mathrm{H})} \approx 0 \mathrm{~Hz}$ and further confirmed by NOESY spectrum. Homochiral $(S)$-proline was also examined, but the enantioselectivity of the reaction was low $\left(23 \%\right.$ ee, determined by chiral HPLC analysis). ${ }^{12}$ Proline methyl ester combined with NaOAc gave 3a in lower yield (Table 1, entry 15). The result suggests that the free carboxylic group of proline is important for the catalytic activity. The hypothesis was further supported by the fact that pyrrolidine combined with NaOAc gave moderated yield of $\mathbf{3 a}$ (Table 1, entry 16). 
Table 1. Reaction of 1a and 2a in the presence of bases ${ }^{\mathrm{a}}$

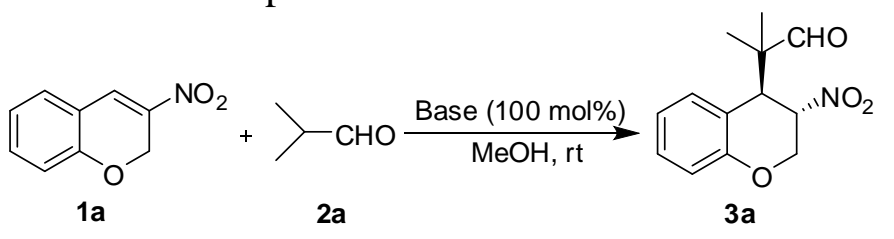

\begin{tabular}{lll}
\hline Entry & Base & Yield $(\%)^{\text {b,c }}$ \\
\hline 1 & $\mathrm{NaOAc}_{2}$ & 0 \\
2 & $\mathrm{~K}_{2} \mathrm{CO}_{3}$ & 0 \\
3 & $\mathrm{LiOH}$ & 14 \\
4 & $\mathrm{KOH}$ & 13 \\
5 & $\mathrm{TEA}$ & 19 \\
6 & DIPEA & 17 \\
7 & DABCO & 14 \\
8 & DBU & 38 \\
9 & DMAP & 12 \\
10 & NMP & 28 \\
11 & Piperidine & 76 \\
12 & Pyrrolidine & 84 \\
13 & Proline & 28 \\
14 & Proline $(20$ mol $\%) / \mathrm{NaOAc}(100 \mathrm{~mol} \%)$ & 98 \\
15 & Proline methyl ester $(20 \mathrm{~mol} \%) / \mathrm{NaOAc}(100 \mathrm{~mol} \%)$ & 52 \\
16 & Pyrrolidine $(20 \mathrm{~mol} \%) / \mathrm{NaOAc}(100 \mathrm{~mol} \%)$ & 68 \\
\hline
\end{tabular}

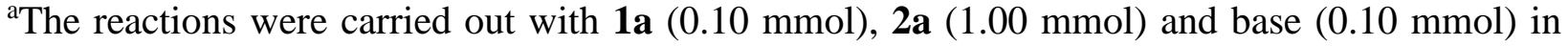
$\mathrm{MeOH}(1.0 \mathrm{~mL})$ at room temperature for $5 \mathrm{~h}$.

${ }^{\mathrm{b}} \mathrm{GC}$ yields determined with dodecane as the internal standard.

'Only trans-3a was observed by NMR analysis.

Using proline/ $\mathrm{NaOAc}$ as the catalyst, a number of reaction solvents were screened and the results are summarized in Table 2. Significant effect of the solvent on the yield was observed. Low yields of 3a were obtained in hexane, toluene, dichloromethane, THF, and diethyl ether (Table 2, entries 1-5). Only traces of product were obtained in 1,4-dioxane and acetonitrile (Table 2, entries 6-7). Methanol was found to be the optimum solvent and excellent yield was achieved (Table 2, entry 8). The result implicates that protic solvents may be necessary for the reaction. 
Table 2. Effect of reaction solvents ${ }^{\mathrm{a}}$

\begin{tabular}{lll}
\hline Entry & Solvent & Yield $(\%)$ \\
\hline 1 & Hexane & 23 \\
2 & Toluene & 12 \\
3 & $\mathrm{CH}_{2} \mathrm{Cl}_{2}$ & 17 \\
4 & $\mathrm{THF}$ & 12 \\
5 & $\mathrm{Et}{ }_{2} \mathrm{O}$ & 17 \\
6 & $1,4-\mathrm{Dioxane}$ & 3 \\
7 & $\mathrm{CH}_{3} \mathrm{CN}$ & 5 \\
8 & $\mathrm{MeOH}$ & 98 \\
\hline
\end{tabular}

${ }^{a}$ The reactions were carried out with $1 \mathrm{a}(0.10 \mathrm{mmol}), \mathbf{2 a}(1.00 \mathrm{mmol}), \mathrm{NaOAc}(0.10 \mathrm{mmol})$ and $(R / S)$-proline $(0.02 \mathrm{mmol})$ in the solvent $(1.0 \mathrm{~mL})$ at room temperature for $5 \mathrm{~h}$.

The effect of the loading of proline and $\mathrm{NaOAc}$ was also studied and the results are summarized in Table 3. The loading of proline and $\mathrm{NaOAc}$ exerted significant effect on the yield. Using $5 \mathrm{~mol} \%$ and $10 \mathrm{~mol} \%$ proline and NaOAc provided poor yields of 3a (Table 3, entries 1-2). Increasing the loading of proline/AcONa to $20 \mathrm{~mol} \%$ improved the yield (Table 3 , entry 3). In addition, increasing the loading of $\mathrm{NaOAc}$ also resulted in better yield (Table 3, entry 4). Excellent yield was achieved using $20 \mathrm{~mol} \%$ proline and $100 \mathrm{~mol} \% \mathrm{NaOAc}$ (Table 3, entry 5).

Table 3. Effect of the loading of proline and $\mathrm{NaOAc}^{\mathrm{a}}$

\begin{tabular}{llll}
\hline Entry & Proline $(\mathrm{mol} \%)$ & $\mathrm{NaOAc}(\mathrm{mol} \%)$ & Yield $(\%)$ \\
\hline 1 & 5 & 5 & 8 \\
2 & 10 & 10 & 17 \\
3 & 20 & 20 & 53 \\
4 & 20 & 50 & 82 \\
5 & 20 & 100 & 98 \\
\hline
\end{tabular}

${ }^{a}$ The reactions were carried out with $\mathbf{1 a}(0.1 \mathrm{mmol}), \mathbf{2 a}(1.0 \mathrm{mmol})$ in $\mathrm{MeOH}(1.0 \mathrm{~mL})$ at room temperature for $5 \mathrm{~h}$.

A mechanism of proline/NaOAc catalysis is suggested (Scheme 1). Enamine intermediate $\mathbf{A}$ is generated from the reaction of proline and isobutyraldehyde. 3-Nitro- $2 \mathrm{H}$-chromene 1a is activated via the hydrogen-bonding interaction with the carboxylic group of proline and the transition state $\mathbf{B}$ is provided. The consequent conjugate addition affords the intermediate $\mathbf{C}$, which is hydrolyzed to provide product 3a and to regenerate proline. The hydrogen-bonding interaction in the transition state $\mathbf{B}$ seems to be important for the activation of 3-nitro- $2 \mathrm{H}$ chromene 3a, since the pyrrolidine and proline methyl ester gave much lower yield than proline. 
Although AcONa was found to be very important for achieving good yield, its exact function in the reaction is not clear. It may work as a base to promote the formation of enamine intermediate A.

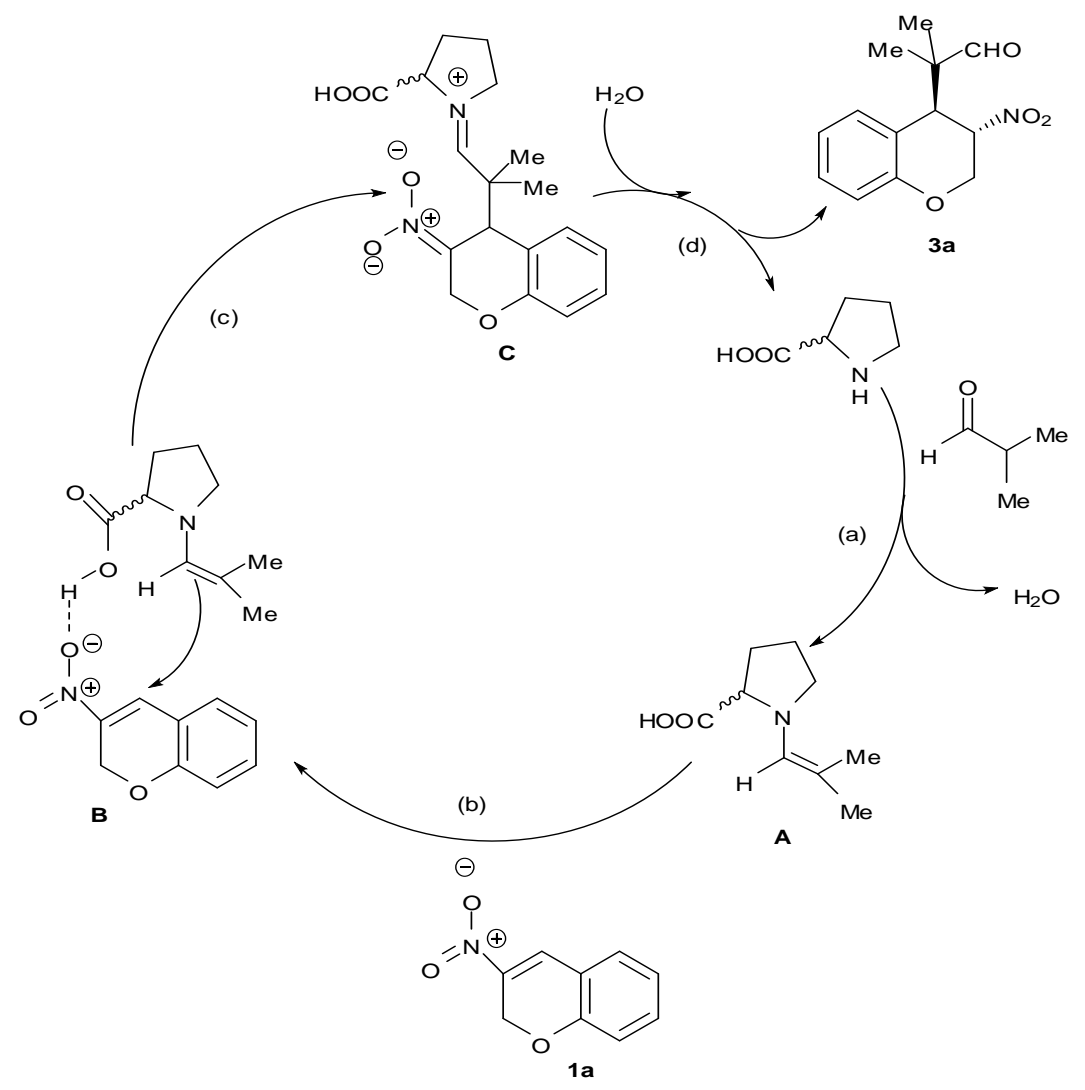

\section{Scheme 1}

A variety of 3-nitro- $2 H$-chromenes $\mathbf{1 a - 1 j}$ were examined in the reaction with isobutyraldehyde 2a. The results are summarized in Table 4. The substitutionof the aromatic ring by a methoxy group at the C7 or C8 position was tolerated very well (Table 4, entries 2-3). Substitution of the aromatic ring at $\mathrm{C} 6$ with methoxy, methyl, or a halogen also provided the products in good yields (Table 4, entries 4-7). However the substitution at C6 with a nitro group or the disubstitution at C6 and C8 with an halogen resulted in low yields (Table 4, entries 8-10). In these cases, some unidentified side products were observed. 
Table 4. Reactions of isobutyraldehyde $\mathbf{2 a}$ with a variety of 3-nitro- $2 H$-chromenes $\mathbf{1 a}-\mathbf{1} \mathbf{j}^{\mathbf{a}}$

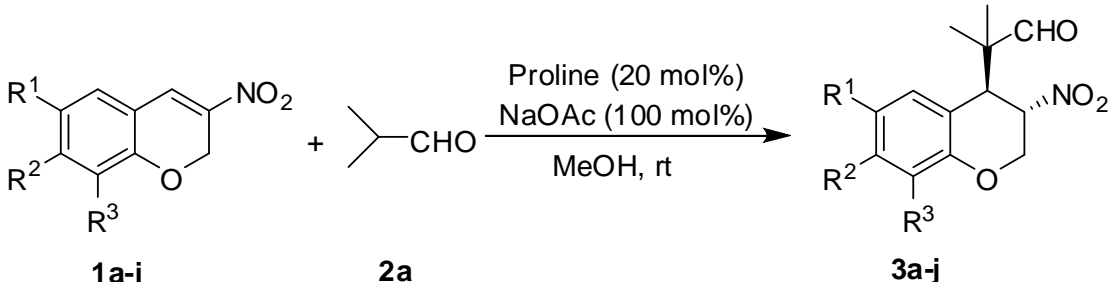

\begin{tabular}{|c|c|c|c|c|c|c|}
\hline \multirow[t]{2}{*}{ Entry } & \multicolumn{3}{|c|}{ Substrate } & \multirow[t]{2}{*}{ Product } & \multirow[t]{2}{*}{ Time (h) } & \multirow[t]{2}{*}{ Yield $(\%)^{\mathrm{b}, \mathrm{c}}$} \\
\hline & $\mathrm{R}^{1}$ & $\mathrm{R}^{2}$ & $\mathrm{R}^{3}$ & & & \\
\hline 1 & $\mathrm{H}$ & $\mathrm{H}$ & $\mathrm{H}$ & $\mathbf{3 a}$ & 5 & 96 \\
\hline 2 & $\mathrm{H}$ & $\mathrm{H}$ & $\mathrm{CH}_{3} \mathrm{O}$ & $3 \mathbf{b}$ & 6 & 92 \\
\hline 3 & $\mathrm{H}$ & $\mathrm{CH}_{3} \mathrm{O}$ & $\mathrm{H}$ & $3 c$ & 6 & 89 \\
\hline 4 & $\mathrm{CH}_{3} \mathrm{O}$ & $\mathrm{H}$ & $\mathrm{H}$ & $3 d$ & 6 & 85 \\
\hline 5 & $\mathrm{CH}_{3}$ & $\mathrm{H}$ & $\mathrm{H}$ & $3 \mathbf{e}$ & 8 & 71 \\
\hline 6 & $\mathrm{Cl}$ & $\mathrm{H}$ & $\mathrm{H}$ & 3f & 3 & 77 \\
\hline 7 & $\mathrm{Br}$ & $\mathrm{H}$ & $\mathrm{H}$ & $3 \mathbf{g}$ & 2 & 81 \\
\hline 8 & $\mathrm{NO}_{2}$ & $\mathrm{H}$ & $\mathrm{H}$ & $3 \mathbf{h}$ & 3.5 & 20 \\
\hline 9 & $\mathrm{Cl}$ & $\mathrm{H}$ & $\mathrm{Cl}$ & $3 \mathbf{i}$ & 2.5 & 40 \\
\hline 10 & $\mathrm{Br}$ & $\mathrm{H}$ & $\mathrm{Br}$ & $\mathbf{3 j}$ & 4 & 31 \\
\hline
\end{tabular}

${ }^{\mathrm{a}}$ The reactions were carried out with $\mathbf{1 a - 1 j}(0.10 \mathrm{mmol}), \mathbf{2 a}(1.00 \mathrm{mmol}),(R / S)$-proline $(0.02 \mathrm{mmol})$ and $\mathrm{NaOAc}(0.10 \mathrm{mmol})$ in $\mathrm{MeOH}(1.0 \mathrm{~mL})$ at room temperature.

b Isolated yields.

${ }^{\mathrm{c} O n l y}$ trans-products were observed by NMR analysis.

A number of aldehydes and ketones were also examined and the results are summarized in Table 5. Acetaldehyde provided the product in low yield after extended reaction time (Table 5, entry 2). Propanal and butanal were highly reactive substrates and the reactions could be completed in less than $5 \mathrm{~min}$. The corresponding adducts were obtained in good yields, but with low diastereoselectivities (Table 5, entries 3-4). Cyclopentane carbaldehyde also provided product 4e in good yield (Table 5, entry 5). Cyclopentanone was found to be a good substrate for the transformation and good yield could be achieved (Table 5, entry 6). Cyclohexanone gave lower yield than cyclopentanone, probably due to bigger steric hindrance (Table 5, entry 7). Acetone and acetophenone provided products $\mathbf{4 h}$ and $\mathbf{4 i}$ respectively in moderate yields (Table 5, entries 8-9). 1-Methoxyl acetone gave product $\mathbf{4 j}$ in good yield and with complete regioselectivity, but the diastereoselectivity was low (Table 5, entry 10). Hydroxylacetone also afforded the corresponding products, but in moderate yield (Table 5, entry 11). 
Table 5. Reaction of $\mathbf{1 a}$ with aldehydes and ketones $\mathbf{2}^{\mathrm{a}}$

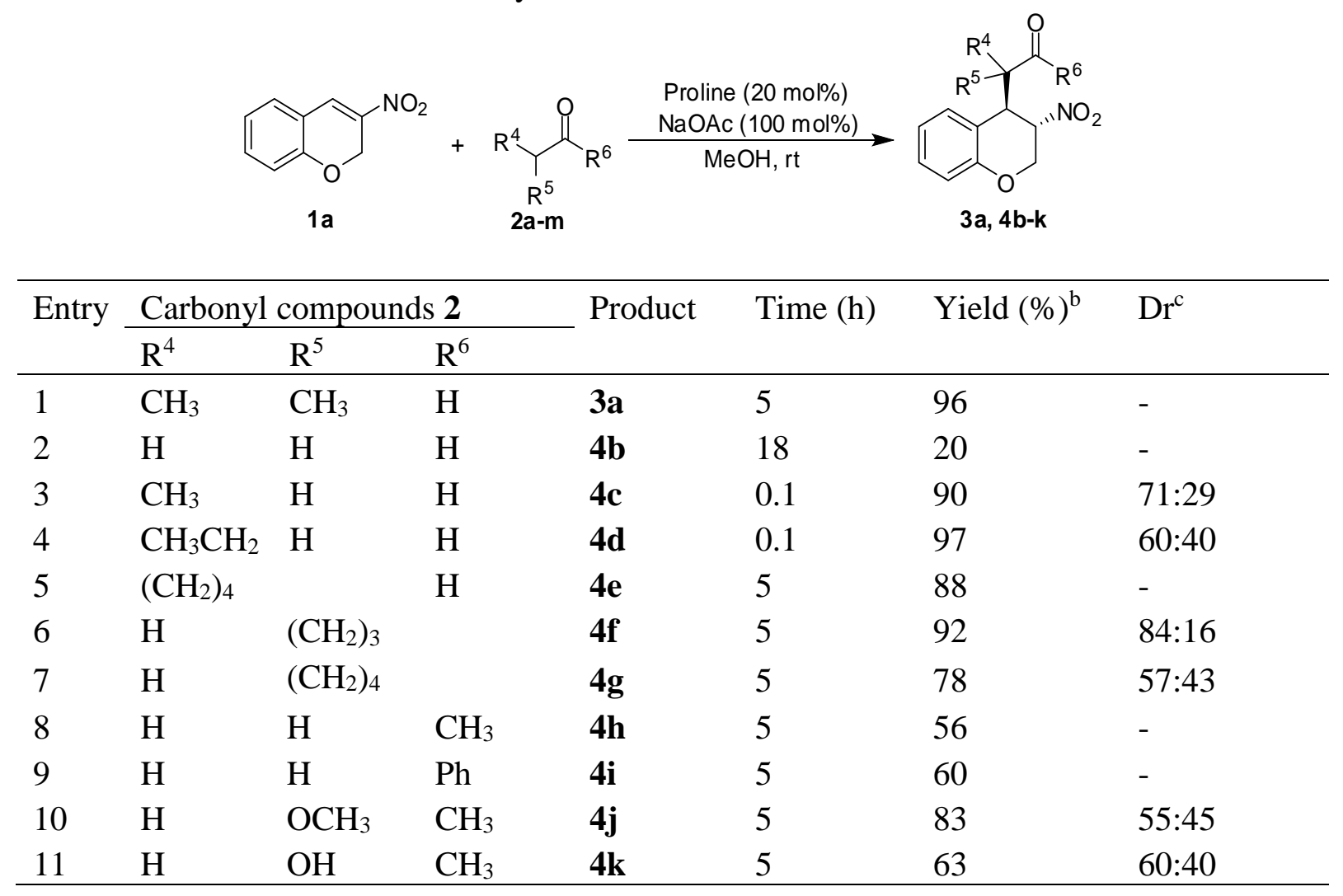

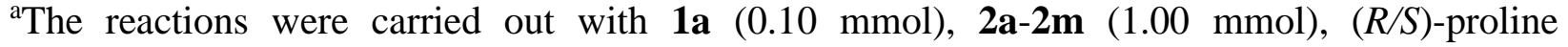
$(0.02 \mathrm{mmol})$ and $\mathrm{NaOAc}(0.10 \mathrm{mmol})$ in $\mathrm{MeOH}(1.0 \mathrm{~mL})$ at room temperature.

${ }^{\mathrm{b}}$ Isolated yields.

'Determined by NMR analysis.

Product 3a could be reduced with zinc powder under acid conditions and chroman[3,4$b$ ]pyrrole 5 was obtained in excellent yield (Scheme 2). The 3,4-cis-configuration was established by NOE analysis as well as the coupling constant ${ }^{3} J_{(3-\mathrm{H}, 4-\mathrm{H})}=11.8 \mathrm{~Hz}$. The racemization at $\mathrm{C}_{3}$ of $\mathbf{3 a}$ occurred under the reaction conditions and thus the thermodynamically favoured 5 showing the cis-fusion was obtained.

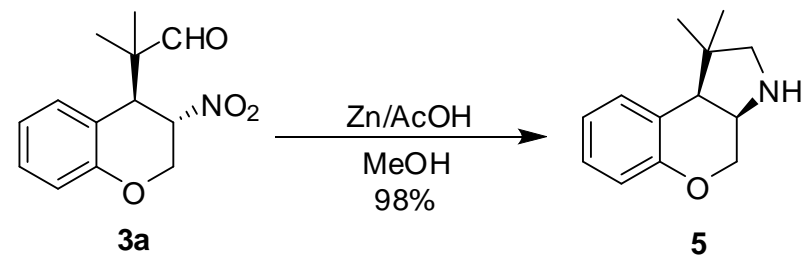

Scheme 2 


\section{Conclusions}

In conclusion, we have developed an efficient conjugate addition of aldehydes and ketones to 3 nitro- $2 H$-chromenes. Proline associated with $\mathrm{NaOAc}$ was found to be the best catalyst. An enamine activation mechanism was proposed. The reaction provided a number of 4-substituted 3-nitrochromans, sometimes in good yields and with excellent diastereoselectivities. The reduction of trans-2-methyl-2-(3-nitrochroman-4-yl)propanal 3a with zinc/AcOH gave the chroman[3,4-b]pyrrole 5 in one step. Further studies are currently underway to apply the method for the synthesis of valuable chromane derivatives and to develop an enantioselective version of the reaction.

\section{Experimental Section}

General. All solvents were used as commercial anhydrous grade without further purification. Nitroethylene was purchased from Taiyang Chemical Company of China. The flash column chromatography was carried out over silica gel (230-400 mesh), purchased from Qingdao Haiyang Chemical Company. Melting points were recorded on an electrothermal digital meltingpoint apparatus. ${ }^{1} \mathrm{H}$ and ${ }^{13} \mathrm{C}$ NMR spectra were recorded on a Bruker Avance $400 \mathrm{MHz}$ spectrometer as solutions in $\mathrm{CDCl}_{3}$ unless otherwise stated. Chemical shifts in ${ }^{1} \mathrm{H}$ NMR spectra are reported in parts per million $\left(\mathrm{ppm}, \delta\right.$ ) downfield from the internal standard $\mathrm{Me}_{4} \mathrm{Si}$ (TMS, $\delta=$ $0.00 \mathrm{ppm}$ ). Chemical shifts in ${ }^{13} \mathrm{C}$ NMR spectra are reported relative to the central line of the chloroform signal $(\delta=77.0 \mathrm{ppm})$. High-resolution mass spectra were obtained with the Thermo MAT 95XP mass spectrometer. The low resolution mass spectra were obtained with the Thermo Trace GC Ultra - DSQ II and Agilent 6120 (Quadrupole LC-MS) mass spectrometer. Infrared (IR) spectra were recorded on a Bruker Tensor 37 spectrophotometer. Data are represented as frequency of absorption $\left(\mathrm{cm}^{-1}\right)$. Enantiomeric excesses of compound 3a were determined by HPLC using a Daicel Chiralpak AD-H column (hexane $/ i-\mathrm{PrOH}=95 / 5, \lambda=210 \mathrm{~nm}$, $0.5 \mathrm{~mL} / \mathrm{min}$ ). GC yields were determined by GC (Agilent $6890 \mathrm{~N}$ ) with a Agilent capillary column HP-5 $(30 \mathrm{~m} \times 0.32 \mathrm{~mm}, 0.25 \mu \mathrm{m})$ and dodecane was used as the internal standard.

\section{Typical experimental procedure for the synthesis of 3-nitro- $2 \mathrm{H}$-chromenes $1 \mathrm{a}-1 \mathrm{j}^{\mathrm{8a}, \mathrm{b}}$}

A solution of salicylaldehyde $(1.22 \mathrm{~g}, 10 \mathrm{mmol})$ and $\mathrm{Bu}_{2} \mathrm{NH}(0.65 \mathrm{~g}, 5 \mathrm{mmol})$ in dry chloroform $(80 \mathrm{~mL})$ was placed in a round-bottomed flask fitted with a Dean-Stark apparatus. At the room temperature, nitroethylene $(0.73 \mathrm{~g}, 10 \mathrm{mmol})$ was added slowly with a syringe pump over $24 \mathrm{~h}$. Then the reaction mixture was refluxed for $24 \mathrm{~h}$ and the water formed during the reaction was removed by the Dean-Stark apparatus. A second portion of nitroethylene $(0.73 \mathrm{~g}, 10 \mathrm{mmol})$ was added according to the above procedure and the reaction solution was refluxed for further $24 \mathrm{~h}$. After cooling down, the reaction solution was filtered through a layer of silica gel and washed with $\mathrm{CH}_{2} \mathrm{Cl}_{2}$ /petroleum ether $(V / V=1 / 1)$. The filtrate was washed with $1 \%$ aqueous sodium 
hydroxide to remove unreacted salicylaldehyde, and then with water. The organic layer was dried over anhydrous $\mathrm{MgSO}_{4}$ and evaporated under vacuum. The residue was recrystallized from hexane to give 3-nitro- $2 H$-chromene $\mathbf{1 a}$.

3-Nitro-2H-chromene (1a). ${ }^{8 a, e}$ Yield: $45 \%$. yellow solid, Mp 77.0-78.0 ${ }^{\circ} \mathrm{C}$ (lit. ${ }^{8 a} \mathrm{Mp} 79.5-$ $\left.80.5{ }^{\circ} \mathrm{C}\right) .{ }^{1} \mathrm{H}$ NMR $\left(400 \mathrm{MHz}, \mathrm{CDCl}_{3}\right): \delta=7.78(\mathrm{~s}, 1 \mathrm{H}), 7.34(\mathrm{~m}, 1 \mathrm{H}), 7.25(\mathrm{dd}, J=7.6,1.6 \mathrm{~Hz}$, $1 \mathrm{H}), 7.00(\mathrm{~m}, 1 \mathrm{H}), 6.91(\mathrm{~d}, J=8.2 \mathrm{~Hz}, 1 \mathrm{H}), 5.25(\mathrm{~d}, J=1.1 \mathrm{~Hz}, 2 \mathrm{H}) ;{ }^{13} \mathrm{C}$ NMR $(100 \mathrm{MHz}$, $\left.\mathrm{CDCl}_{3}\right): \delta=154.95,139.15,133.96,130.53,129.21,122.70,118.27,116.58,62.93$; IR (KBr): 1651, 1601, 1570, 1481, 1320, 1226, 1198, 1123, 1072, 764, $713 \mathrm{~cm}^{-1}$; MS (ESI): $\mathrm{m} / \mathrm{z}=176.0$ [M - H] ; HRMS (ESI) calcd for $\mathrm{C}_{9} \mathrm{H}_{6} \mathrm{NO}_{3}{ }^{-}[\mathrm{M}-\mathrm{H}]^{-}: 176.0348$, found: 176.0360 .

8-Methoxy-3-nitro-2H-chromene (1b). ${ }^{8 \mathrm{a}, \mathrm{b}, \mathrm{e}}$ Yield: 55\%. Yellow solid, Mp 125.6-127.1 ${ }^{\circ} \mathrm{C}$ (lit. ${ }^{8 \mathrm{a}}$ Mp 128-129 $\left.{ }^{\circ} \mathrm{C}\right) .{ }^{1} \mathrm{H}$ NMR (400 MHz, $\left.\mathrm{CDCl}_{3}\right): \delta=7.78(\mathrm{~s}, 1 \mathrm{H}), 6.98(\mathrm{~m}, 2 \mathrm{H}), 6.89$ (dd, $J=6.5$, $2.6 \mathrm{~Hz}, 1 \mathrm{H}), 5.31(\mathrm{~s}, 2 \mathrm{H}), 3.90(\mathrm{~s}, 3 \mathrm{H}) ;{ }^{13} \mathrm{C} \mathrm{NMR}\left(100 \mathrm{MHz}, \mathrm{CDCl}_{3}\right): \delta=148.22,143.92,139.29$, 129.24, 122.60, 122.24, 119.03, 116.40, 63.20, 56.26; IR (KBr): 1577, 1559, 1541, 1508, 1480, 1338, 1272, 1015, 784, $719 \mathrm{~cm}^{-1}$; MS (ESI): $\mathrm{m} / z=206.1[\mathrm{M}-\mathrm{H}]^{-}$; HRMS (ESI) calcd for $\mathrm{C}_{10} \mathrm{H}_{8} \mathrm{NO}_{4}^{-}[\mathrm{M}-\mathrm{H}]^{-}:$206.0453, found: 206.0462 .

7-Methoxy-3-nitro-2H-chromene (1c). ${ }^{8 \mathrm{a}, \mathrm{b}, \mathrm{e}}$ Yield: $50 \%$. Yellow solid, Mp 117.6-118.8 ${ }^{\circ} \mathrm{C}$ (lit. $\left.{ }^{8 \mathrm{a}} \mathrm{Mp} 120-121{ }^{\circ} \mathrm{C}\right) .{ }^{1} \mathrm{H} \mathrm{NMR}\left(400 \mathrm{MHz} \mathrm{CDCl}_{3}\right): \delta=7.78(\mathrm{~s}, 1 \mathrm{H}), 7.17(\mathrm{~d}, J=8.5 \mathrm{~Hz}, 1 \mathrm{H})$, $6.57(\mathrm{dd}, J=8.5,2.4 \mathrm{~Hz}, 1 \mathrm{H}), 6.45(\mathrm{~d}, J=2.4 \mathrm{~Hz}, 1 \mathrm{H}), 5.24(\mathrm{~s}, 2 \mathrm{H}), 3.83(\mathrm{~s}, 3 \mathrm{H}) ;{ }^{13} \mathrm{C} \mathrm{NMR}(100$ $\left.\mathrm{MHz}, \mathrm{CDCl}_{3}\right): \delta=164.84,156.95,136.18,131.80,129.76,111.34,109.64,101.80,63.14,55.68$; IR (KBr): 1618, 1559, 1508, 1490, 1459, 1437, 1337, 1257, 1077, 954, 870, $715 \mathrm{~cm}^{-1}$; MS (ESI): $m / z=206.0[\mathrm{M}-\mathrm{H}]^{-}$; HRMS (ESI) calcd for $\mathrm{C}_{10} \mathrm{H}_{8} \mathrm{NO}_{4}^{-}[\mathrm{M}-\mathrm{H}]^{-}: 206.0453$, found: 206.0460.

6-Methoxy-3-nitro-2H-chromene (1d). ${ }^{8 \mathrm{a}, \mathrm{b}, \mathrm{e}}$ Yield: $56 \%$; Yellow solid, Mp 88.7-89.8 ${ }^{\circ} \mathrm{C}_{\text {(lit. }}{ }^{8 \mathrm{a}}$ Mp 90-91 ${ }^{\circ} \mathrm{C}$ ). ${ }^{1} \mathrm{H}$ NMR (400 MHz, $\left.\mathrm{CDCl}_{3}\right): \delta=7.71(\mathrm{~s}, 1 \mathrm{H}), 6.90$ (q, $\left.J=8.9,2.9 \mathrm{~Hz}, 1 \mathrm{H}\right), 6.84$ $(\mathrm{d}, J=8.9 \mathrm{~Hz}, 1 \mathrm{H}), 6.75(\mathrm{~d}, J=2.9 \mathrm{~Hz}, 1 \mathrm{H}), 5.16(\mathrm{~d}, J=0.8 \mathrm{~Hz}, 2 \mathrm{H}), 3.78(\mathrm{~s}, 3 \mathrm{H}) ;{ }^{13} \mathrm{C} \mathrm{NMR}$ (100 MHz, $\mathrm{CDCl}_{3}$ ): $\delta=154.91,148.90,139.81,129.22,120.04,118.84,117.29,114.02,62.88$, 55.80; IR (KBr): 3060, 1652, 1576, 1507, 1489, 1458, 1320, 1255, 1135, $842 \mathrm{~cm}^{-1}$; MS (ESI): $m / z=206.1[\mathrm{M}-\mathrm{H}]^{-}$; HRMS (ESI) calcd for $\mathrm{C}_{10} \mathrm{H}_{8} \mathrm{NO}_{4}^{-}[\mathrm{M}-\mathrm{H}]^{-}: 206.0453$, found: 206.0459 .

6-Methyl-3-nitro-2H-chromene (1e). ${ }^{8 b, e}$ Yield: $42 \%$. Yellow solid, Mp 74.1-75.6 ${ }^{\circ} \mathrm{C}$ (lit. ${ }^{8 \mathrm{~b}} \mathrm{Mp}$ 75-76 $\left.{ }^{\circ} \mathrm{C}\right) .{ }^{1} \mathrm{H}$ NMR $\left(400 \mathrm{MHz}, \mathrm{CDCl}_{3}\right): \delta 7.75(\mathrm{~s}, 1 \mathrm{H}), 7.14(\mathrm{dd}, J=8.3,1.9 \mathrm{~Hz}, 1 \mathrm{H}), 7.05(\mathrm{~d}, J$ $=1.4 \mathrm{~Hz}, 1 \mathrm{H}), 6.82(\mathrm{dd}, J=8.3,3.8 \mathrm{~Hz}, 1 \mathrm{H}), 5.22(\mathrm{~d}, J=0.9 \mathrm{~Hz}, 2 \mathrm{H}), 2.29(\mathrm{~s}, 3 \mathrm{H}) ;{ }^{13} \mathrm{C}$ NMR $\left(100 \mathrm{MHz}, \mathrm{CDCl}_{3}\right): \delta=152.89,139.18,134.70,132.21,130.63,129.45,118.13,116.30,62.93$, 20.36; IR (KBr): 2920, 1576, 1508, 1340, 1158, 1076, 945, $860 \mathrm{~cm}^{-1}$; MS (ESI): $m / z=190.0$ [M - H] $]^{-}$; HRMS (ESI) calcd for $\mathrm{C}_{10} \mathrm{H}_{8} \mathrm{NO}_{3}{ }^{-}[\mathrm{M}-\mathrm{H}]^{-}: 190.0504$, found: 190.0507.

6-Chloro-3-nitro-2H-chromene (1f). ${ }^{8 \mathrm{a}}$ Yield: $46 \%$. Yellow solid, Mp 109.5-110.4 ${ }^{\circ} \mathrm{C}$ (lit. ${ }^{8 \mathrm{a}} \mathrm{Mp}$ 115-116 ${ }^{\circ} \mathrm{C}$ ). ${ }^{1} \mathrm{H}$ NMR (400 MHz, $\left.\mathrm{CDCl}_{3}\right): \delta=7.70(\mathrm{~s}, 1 \mathrm{H}), 7.28(\mathrm{dd}, J=8.6,2.5 \mathrm{~Hz}, 1 \mathrm{H}), 7.23$ $(\mathrm{d}, J=2.5 \mathrm{~Hz}, 1 \mathrm{H}), 6.86(\mathrm{~d}, J=8.6 \mathrm{~Hz}, 1 \mathrm{H}), 5.25(\mathrm{~d}, J=1.1 \mathrm{~Hz}, 2 \mathrm{H}) ;{ }^{13} \mathrm{C} \mathrm{NMR}(100 \mathrm{MHz}$, $\left.\mathrm{CDCl}_{3}\right): \delta=153.33,140.07,133.41,129.53,127.84,127.59,119.51,117.92,63.05$; IR $(\mathrm{KBr})$ : $3080,1655,1517,1479,1338,1163,1070,951,861,840,646 \mathrm{~cm}^{-1}$; MS (ESI): $\mathrm{m} / \mathrm{z}=210.0[\mathrm{M}-$ $\mathrm{H}]^{-}$; HRMS (ESI) calcd for $\mathrm{C}_{9} \mathrm{H}_{5} \mathrm{ClNO}_{3}^{-}[\mathrm{M}-\mathrm{H}]^{-}: 209.9958$, found: 210.0002 . 
6-Bromo-3-nitro-2H-chromene(1g). ${ }^{8 a, b}$ Yield: 40\%. Yellow solid, Mp 120.1-121.5 ${ }^{\circ} \mathrm{C}$ (lit. ${ }^{8 \mathrm{a}}$ Mp 121-122 $\left.{ }^{\circ} \mathrm{C}\right) .{ }^{1} \mathrm{H}$ NMR (400 MHz, $\left.\mathrm{CDCl}_{3}\right): \delta=7.70(\mathrm{~s}, 1 \mathrm{H}), 7.42(\mathrm{dd}, J=8.6,2.4 \mathrm{~Hz}, 1 \mathrm{H})$, $7.38(\mathrm{~d}, J=2.4 \mathrm{~Hz}, 1 \mathrm{H}), 6.81(\mathrm{~d}, J=8.6 \mathrm{~Hz}, 1 \mathrm{H}), 5.26(\mathrm{~d}, J=1.1 \mathrm{~Hz}, 2 \mathrm{H}) ;{ }^{13} \mathrm{C} \mathrm{NMR}(100 \mathrm{MHz}$, $\left.\mathrm{CDCl}_{3}\right): \delta=153.86,139.98,136.33,132.49,127.74,120.03,118.33,114.62,63.03$; IR (KBr): 1513, 1474, 1331, 1013, 901, 836, $709 \mathrm{~cm}^{-1}$; MS (ESI): $\mathrm{m} / \mathrm{z}=253.8$ [M - H] $]^{-}$HRMS (ESI) calcd for $\mathrm{C}_{9} \mathrm{H}_{5} \mathrm{BrNO}_{3}{ }^{-}[\mathrm{M}-\mathrm{H}]^{-}: 253.9453$, found: 253.9460 .

3,6-Dinitro-2H-chromene(1h). ${ }^{8 \mathrm{a}}$ Yield: $58 \%$. Yellow solid, Mp 157.0-158.0 ${ }^{\circ} \mathrm{C}$ (lit. ${ }^{8 \mathrm{a}} \mathrm{Mp} 160$ $\left.161{ }^{\circ} \mathrm{C}\right) .{ }^{1} \mathrm{H} \mathrm{NMR}\left(400 \mathrm{MHz}, \mathrm{CDCl}_{3}\right): \delta=8.19-8.24(\mathrm{~m}, 2 \mathrm{H}), 7.81(\mathrm{~s}, 1 \mathrm{H}), 7.02(\mathrm{~d}, J=8.9 \mathrm{~Hz}$, $1 \mathrm{H}), 5.41(\mathrm{~d}, J=1.2 \mathrm{~Hz}, 2 \mathrm{H}) ;{ }^{13} \mathrm{C} \mathrm{NMR}\left(100 \mathrm{MHz}, \mathrm{CDCl}_{3}\right): \delta 159.36,142.78,140.39,129.03$, 126.95, 125.91, 118.02, 117.17, 63.79; IR (KBr): 3068, 1660, 1661, 1509, 1480, 1456, 1332 , 1237, 1209, 1101, 837, $752 \mathrm{~cm}^{-1}$; MS (ESI): $\mathrm{m} / \mathrm{z}=221.0[\mathrm{M}-\mathrm{H}]^{-}$; HRMS (ESI) calcd for $\mathrm{C}_{9} \mathrm{H}_{5} \mathrm{~N}_{2} \mathrm{O}_{5}{ }^{-}[\mathrm{M}-\mathrm{H}]^{-}: 221.0198$, found: 221.0202 .

6,8-Dichloro-3-nitro-2H-chromene (1i). Yield: 54\%. Yellow solid, Mp 130.2-131.1 ${ }^{\circ} \mathrm{C}$. ${ }^{1} \mathrm{H}$ NMR (400 MHz, $\left.\mathrm{CDCl}_{3}\right): \delta=7.68(\mathrm{~s}, 1 \mathrm{H}), 7.39(\mathrm{t}, J=2.7 \mathrm{~Hz}, 1 \mathrm{H}), 7.16(\mathrm{~d}, J=2.1 \mathrm{~Hz}, 1 \mathrm{H}), 5.35$ $(\mathrm{s}, 2 \mathrm{H}) ;{ }^{13} \mathrm{C} \mathrm{NMR}\left(100 \mathrm{MHz}, \mathrm{CDCl}_{3}\right): \delta=149.13,140.57,133.44,128.07,127.46,127.15$, 122.85, 120.35, 63.58; IR (KBr): 1558, 1508, 1457, 1326, 1163, 1071, 867, $710 \mathrm{~cm}^{-1}$; MS (ESI): $m / z=243.8[\mathrm{M}-\mathrm{H}]^{-}$; HRMS (ESI) calcd for $\mathrm{C}_{9} \mathrm{H}_{4} \mathrm{Cl}_{2} \mathrm{NO}_{3}^{-}[\mathrm{M}-\mathrm{H}]^{-}: 243.9568$, found: 243.9555 .

6,8-Dibromo-3-nitro-2H-chromene (1j). ${ }^{8 \mathrm{a}}$ Yield: $64 \%$. Yellow solid, Mp 142.6-143.8 ${ }^{\circ} \mathrm{C}\left(\right.$ lit. $^{8 \mathrm{a}}$ Mp 146.5-147.5 $\left.{ }^{\circ} \mathrm{C}\right) .{ }^{1} \mathrm{H}$ NMR (400 MHz, $\left.\mathrm{CDCl}_{3}\right): \delta=7.67(\mathrm{~m}, 2 \mathrm{H}), 7.33(\mathrm{~d}, J=2.3 \mathrm{~Hz}, 1 \mathrm{H})$, $5.36(\mathrm{~d}, J=1.2 \mathrm{~Hz}, 2 \mathrm{H}) ;{ }^{13} \mathrm{C} \mathrm{NMR}\left(100 \mathrm{MHz}, \mathrm{CDCl}_{3}\right): \delta 150.65,140.40,138.88,131.65$, 127.03, 120.66, 114.55, 111.50, 63.65; IR (KBr): 1541, 1507, 1458, 1330, $1190 \mathrm{~cm}^{-1}$; MS (ESI): $m / z=331.8[\mathrm{M}-\mathrm{H}]^{-}$; HRMS (ESI) calcd for $\mathrm{C}_{9} \mathrm{H}_{4} \mathrm{Br}_{2} \mathrm{NO}_{3}{ }^{-}[\mathrm{M}-\mathrm{H}]^{-}: 331.8558$, found: 331.8415 .

Typical experimental procedure for the conjugate addition of carbonyl compounds to 3nitro-2H-chromenes

A mixture of proline $(2.30 \mathrm{mg}, 0.02 \mathrm{mmol}), \mathrm{NaOAc}(8.20 \mathrm{mg}, 0.10 \mathrm{mmol})$ and 3-nitro-2Hchromene 1a $(17.72 \mathrm{mg}, 0.10 \mathrm{mmol})$ in $\mathrm{MeOH}(1.0 \mathrm{~mL})$ was stirred for $5 \mathrm{~min}$ at room temperature. Then isobutyraldehyde $2 \mathbf{a}(72.11 \mathrm{mg}, 1.00 \mathrm{mmol})$ was added and the reaction mixture was stirred for $5 \mathrm{~h}$ at room temperature. After the solvent was evaporated under vacuum, the residue was purified by flash column chromatography over silica gel (EtOAc/petroleum ether) to provide $\mathbf{3 a}$.

trans-2-(3-Nitrochroman-4-yl)-2-methylpropanal (3a). Yellow oil. ${ }^{1} \mathrm{H}$ NMR (400 MHz, $\left.\mathrm{CDCl}_{3}\right): \delta=9.53(\mathrm{~s}, 1 \mathrm{H}), 7.20(\mathrm{~m}, 1 \mathrm{H}), 7.06(\mathrm{dd}, J=7.7,1.4 \mathrm{~Hz}, 1 \mathrm{H}), 6.93(\mathrm{~m}, 2 \mathrm{H}), 4.97(\mathrm{~m}, 1 \mathrm{H})$, 4.64 (ddd, $J=12.8,4.33,1.53 \mathrm{~Hz}, 1 \mathrm{H}), 4.45$ (dd, $\mathrm{J}=12.8,5.0 \mathrm{~Hz}, 1 \mathrm{H}), 4.03$ (s, 1H), 1.16 (s, $3 \mathrm{H}), 1.13(\mathrm{~s}, 3 \mathrm{H}) ;{ }^{13} \mathrm{C}$ NMR $\left(100 \mathrm{MHz}, \mathrm{CDCl}_{3}\right): \delta=203.93,154.43,130.45,129.35,121.88$, 118.45, 117.44, 80.88, 64.99, 49.71, 43.16, 20.50, 20.32; IR (KBr): 2968, 2918, 2850, 1719, 1608, 1585, 1556, 1508, 1490, 1397, 1361, 1230, 1120, 1042, $758 \mathrm{~cm}^{-1}$; MS (ESI): $\mathrm{m} / \mathrm{z}=248.1$ [M - H] ; HRMS (ESI) calcd for $\mathrm{C}_{13} \mathrm{H}_{14} \mathrm{NO}_{4}{ }^{-}[\mathrm{M}-\mathrm{H}]^{-}:$: 248.0923, found: 248.0912 .

trans-2-(8-Methoxy-3-nitrochroman-4-yl)-2-methylpropanal (3b). Yellow oil. ${ }^{1} \mathrm{H}$ NMR (400 $\left.\mathrm{MHz} \mathrm{CDCl}_{3}\right): \delta=9.55(\mathrm{~s}, 1 \mathrm{H}), 6.90(\mathrm{t}, J=8.1 \mathrm{~Hz}, 1 \mathrm{H}), 6.82(\mathrm{dd}, J=8.1,1.0 \mathrm{~Hz}, 1 \mathrm{H}), 6.66(\mathrm{dd}, J$ 
$=7.8,0.9 \mathrm{~Hz}, 1 \mathrm{H}), 4.99(\mathrm{~m}, 1 \mathrm{H}), 4.72(\mathrm{ddd}, J=12.8,4.4,1.4 \mathrm{~Hz}, 1 \mathrm{H}), 4.53(\mathrm{dd}, \mathrm{J}=12.8,5.1 \mathrm{~Hz}$, $1 \mathrm{H}), 4.06(\mathrm{~s}, 1 \mathrm{H}), 3.86(\mathrm{~s}, 3 \mathrm{H}), 1.16(\mathrm{~s}, 3 \mathrm{H}), 1.11(\mathrm{~s}, 3 \mathrm{H}) ;{ }^{13} \mathrm{C} \mathrm{NMR}\left(100 \mathrm{MHz}, \mathrm{CDCl}_{3}\right)$ : $\delta=203.77,148.60,143.79,121.98,121.52,119.50,111.13,80.75,65.42,55.93,49.71,42.82$, 20.47, 20.26; IR (KBr): 2921, 2851, 1717, 1587, 1553, 1458, 1361, 1264, 1224, 1102, 1028, $734 \mathrm{~cm}^{-1}$; MS (ESI): $\mathrm{m} / z=278.1[\mathrm{M}-\mathrm{H}]^{-}$; HRMS (ESI) calcd for $\mathrm{C}_{14} \mathrm{H}_{16} \mathrm{NO}_{5}^{-}[\mathrm{M}-\mathrm{H}]^{-}$: 278.1028, found: 278.1034 .

trans-2-(7-Methoxy-3-nitrochroman-4-yl)-2-methylpropanal (3c). Yellow oil. ${ }^{1} \mathrm{H}$ NMR (400 $\left.\mathrm{MHz} \mathrm{CDCl}_{3}\right): \delta=9.52(\mathrm{~s}, 1 \mathrm{H}), 6.95(\mathrm{~d}, J=8.6 \mathrm{~Hz}, 1 \mathrm{H}), 6.52(\mathrm{dd}, J=8.6,2.6 \mathrm{~Hz}, 1 \mathrm{H}), 6.44(\mathrm{~d}, J$ $=2.6 \mathrm{~Hz}, 2 \mathrm{H}), 4.93(\mathrm{~m}, 1 \mathrm{H}), 4.68(\mathrm{ddd}, J=13.0,3.9,1.7 \mathrm{~Hz}, 1 \mathrm{H}), 4.39(\mathrm{dd}, \mathrm{J}=13.0,4.7 \mathrm{~Hz}$, $1 \mathrm{H}), 3.95(\mathrm{~s}, 1 \mathrm{H}), 3.76(\mathrm{~s}, 1 \mathrm{H}), 1.15(\mathrm{~s}, 3 \mathrm{H}), 1.12(\mathrm{~s}, 3 \mathrm{H}) ;{ }^{13} \mathrm{C} \mathrm{NMR}\left(100 \mathrm{MHz}, \mathrm{CDCl}_{3}\right): \delta=204.22$, $160.41,155.10,131.02$, 109.93, 108.73, 102.07, 80.70, 64.63, 55.30, 49.70, 42.70, 20.49, 20.28; IR (KBr): 2920, 2850, 2718, 1718, 1619, 1555, 1506, 1464, 1361, 1202, 1163, 1031, $752 \mathrm{~cm}^{-1}$; MS (ESI): $m / z=278.1[\mathrm{M}-\mathrm{H}]^{-}$; HRMS (ESI) calcd for $\mathrm{C}_{14} \mathrm{H}_{16} \mathrm{NO}_{5}{ }^{-}[\mathrm{M}-\mathrm{H}]^{-}: 278.1028$, found: 278.1032 .

trans-2-(6-Methoxy-3-nitrochroman-4-yl)-2-methylpropanal (3d). Pale yellow oil. ${ }^{1} \mathrm{H}$ NMR $\left(400 \mathrm{MHz}, \mathrm{CDCl}_{3}\right): \delta=9.54(\mathrm{~s}, 1 \mathrm{H}), 6.85(\mathrm{~d}, J=8.9 \mathrm{~Hz}, 1 \mathrm{H}), 6.77(\mathrm{dd}, J=8.9,2.9 \mathrm{~Hz}, 1 \mathrm{H}), 6.59$ $(\mathrm{d}, J=2.9 \mathrm{~Hz}, 1 \mathrm{H}), 4.97(\mathrm{~m}, 1 \mathrm{H}), 4.42-4.53(\mathrm{~m}, 2 \mathrm{H}), 4.02(\mathrm{~d}, J=2.6 \mathrm{~Hz}, 1 \mathrm{H}), 3.75(\mathrm{~s}, 3 \mathrm{H}), 1.16$ (s, 3H), $1.13(\mathrm{~s}, 3 \mathrm{H}) ;{ }^{13} \mathrm{C}$ NMR $\left(100 \mathrm{MHz}, \mathrm{CDCl}_{3}\right): \delta=203.87,154.30,148.63,119.89,118.15$, 115.12, 115.03, 81.21, 65.66, 55.69, 49.86, 43.46, 20.50, 20.21; IR (KBr): 2920, 2851, 1719, 1555, 1499, 1472, 1361, 1212, 1048, $725 \mathrm{~cm}^{-1}$; MS (ESI): $\mathrm{m} / z=278.1$ [M - H] ; HRMS (ESI) calcd for $\mathrm{C}_{14} \mathrm{H}_{16} \mathrm{NO}_{5}^{-}[\mathrm{M}-\mathrm{H}]^{-}: 278.1028$, found: 278.1035 .

trans-2-(6-Methyl-3-nitrochroman-4-yl)-2-methylpropanal (3e). Pale yellow solid, Mp 121.2$123.0^{\circ} \mathrm{C} .{ }^{1} \mathrm{H}$ NMR $\left(400 \mathrm{MHz}, \mathrm{CDCl}_{3}\right): \delta=9.54(\mathrm{~s}, 1 \mathrm{H}), 7.00(\mathrm{dd}, J=8.3,1.9 \mathrm{~Hz}, 1 \mathrm{H}), 6.84(\mathrm{~d}, J$ $=1.5 \mathrm{~Hz}, 1 \mathrm{H}), 6.79(\mathrm{~d}, J=8.3 \mathrm{~Hz}, 1 \mathrm{H}), 4.96(\mathrm{~m}, 1 \mathrm{H}), 4.59(\mathrm{ddd}, J=12.7,4.52,1.5 \mathrm{~Hz}, 1 \mathrm{H})$, $4.43(\mathrm{dd}, \mathrm{J}=12.7,5.1 \mathrm{~Hz}, 1 \mathrm{H}), 3.97(\mathrm{~s}, 1 \mathrm{H}), 2.26(\mathrm{~s}, 3 \mathrm{H}), 1.16(\mathrm{~s}, 3 \mathrm{H}), 1.12(\mathrm{~s}, 3 \mathrm{H}) ;{ }^{13} \mathrm{C} \mathrm{NMR}$ $\left(100 \mathrm{MHz}, \mathrm{CDCl}_{3}\right): \delta=204.02,152.33,131.27,130.63,130.05,118.29,117.15,81.08,65.14$, 49.70, 43.34, 20.69, 20.55, 20.24; IR (KBr): 2924, 2854, 1720, 1553, 1499, 1459, 1359, 1216, 1032, 882, $817 \mathrm{~cm}^{-1}$; MS (ESI): $\mathrm{m} / z=262.1$ [M - H] $]^{-}$HRMS (ESI) calcd for $\mathrm{C}_{14} \mathrm{H}_{16} \mathrm{NO}_{4}{ }^{-}[\mathrm{M}-$ $\mathrm{H}]^{-}: 262.1079$, found: 262.1083 .

trans-2-(6-Chloro-3-nitrochroman-4-yl)-2-methylpropanal (3f). White solid, Mp 86.6-88.2 ${ }^{\circ} \mathrm{C} .{ }^{1} \mathrm{H}$ NMR $\left(400 \mathrm{MHz}, \mathrm{CDCl}_{3}\right): \delta=9.50(\mathrm{~s}, 1 \mathrm{H}), 7.17(\mathrm{dd}, J=8.7,2.5 \mathrm{~Hz}, 1 \mathrm{H}), 7.07$ (d, $J=2.5$ $\mathrm{Hz}, 1 \mathrm{H}), 6.85(\mathrm{~d}, J=8.7 \mathrm{~Hz}, 1 \mathrm{H}), 4.95(\mathrm{~m}, 1 \mathrm{H}), 4.70(\mathrm{ddd}, J=13.0,3.8,1.7 \mathrm{~Hz}, 1 \mathrm{H}), 4.43$ (dd, $J$ $=13.0,4.9 \mathrm{~Hz}, 1 \mathrm{H}), 4.00(\mathrm{~d}, J=1.9 \mathrm{~Hz}, 1 \mathrm{H}), 1.17(\mathrm{~s}, 3 \mathrm{H}), 1.16(\mathrm{~s}, 3 \mathrm{H}) ;{ }^{13} \mathrm{C} \mathrm{NMR}(100 \mathrm{MHz}$, $\left.\mathrm{CDCl}_{3}\right): \delta=203.40,152.87,130.06,129.49,126.67,119.90,118.82,80.36,64.92,49.49,42.76$, 20.70, 20.48; IR (KBr): 3116, 3039, 2919, 1722, 1556, 1486, 1231, 1024, $735 \mathrm{~cm}^{-1}$; MS (ESI): $m / z=282.0[\mathrm{M}-\mathrm{H}]^{-}$; HRMS (ESI) calcd for $\mathrm{C}_{13} \mathrm{H}_{13} \mathrm{ClNO}_{4}^{-}[\mathrm{M}-\mathrm{H}]^{-}: 282.0533$, found: 282.0539. trans-2-(6-Bromo-3-nitrochroman-4-yl)-2-methylpropanal (3g). Yellow oil. ${ }^{1} \mathrm{H}$ NMR (400 $\left.\mathrm{MHz}_{\mathrm{CDCl}}\right): \delta=9.50(\mathrm{~s}, 1 \mathrm{H}), 7.31(\mathrm{dd}, J=8.7,2.4 \mathrm{~Hz}, 1 \mathrm{H}), 7.21(\mathrm{~d}, J=2.3 \mathrm{~Hz}, 1 \mathrm{H}), 6.79(\mathrm{~d}, J$ $=8.7 \mathrm{~Hz}, 1 \mathrm{H}), 4.94(\mathrm{~m}, 1 \mathrm{H}), 4.71(\mathrm{ddd}, J=13.0,3.8,1.7 \mathrm{~Hz}, 1 \mathrm{H}), 4.42(\mathrm{dd}, \mathrm{J}=13.0,4.8 \mathrm{~Hz}$, 1H), 3.99 (s, 1H), 1.17 (s, 6H); ${ }^{13} \mathrm{C}$ NMR (100 MHz, $\left.\mathrm{CDCl}_{3}\right): \delta=203.38,153.38,132.98,132.41$, 
120.37, 119.24, 113.92, 80.32, 64.83, 49.48, 42.72, 20.74, 20.48 ; IR (KBr): 2921, 2851, 1719, 1552, 1474, 1360, 1230, 1179, 1126, 1026, $816 \mathrm{~cm}^{-1}$; MS (ESI): $\mathrm{m} / z=325.9$ [M - H] ; HRMS (ESI) calcd for $\mathrm{C}_{13} \mathrm{H}_{13} \mathrm{BrNO}_{4}^{-}[\mathrm{M}-\mathrm{H}]^{-}: 326.0028$, found: 326.0033 .

trans-2-(3,6-Dinitrochroman-4-yl)-2-methylpropanal (3h). White solid, Mp 125.0-127.1 ${ }^{\circ} \mathrm{C}$. ${ }^{1} \mathrm{H}$ NMR $\left(400 \mathrm{MHz}, \mathrm{CDCl}_{3}\right): \delta=9.51(\mathrm{~s}, 1 \mathrm{H}), 8.07-8.14(\mathrm{~m}, 2 \mathrm{H}), 7.01(\mathrm{~d}, J=9.0 \mathrm{~Hz}, 1 \mathrm{H}), 4.94-$ $5.02(\mathrm{~m}, 2 \mathrm{H}),, 4.50(\mathrm{dd}, \mathrm{J}=14.0,4.6 \mathrm{~Hz}, 1 \mathrm{H}), 4.10(\mathrm{~s}, 1 \mathrm{H}), 1.23(\mathrm{~s}, 3 \mathrm{H}), 1.22(\mathrm{~s}, 3 \mathrm{H},) ;{ }^{13} \mathrm{C} \mathrm{NMR}$ $\left(100 \mathrm{MHz}, \mathrm{CDCl}_{3}\right): \delta=202.69,158.81,141.86,126.85,125.32,118.10,117.90,79.29,64.70$, 49.21, 42.30, 21.14, 20.48; IR (KBr): 2920, 2851, 1717, 1584, 1553, 1515, 1472, 1339, 1237, 1096, $748 \mathrm{~cm}^{-1}$; MS (ESI): $\mathrm{m} / \mathrm{z}=293.1[\mathrm{M}-\mathrm{H}]^{-}$; HRMS (ESI) calcd for $\mathrm{C}_{13} \mathrm{H}_{13} \mathrm{NO}_{6}{ }^{-}[\mathrm{M}-\mathrm{H}]^{-}$: 293.0774, found: 293.0771 .

trans-2-(6,8-Dichloro-3-nitrochroman-4-yl)-2-methylpropanal (3i). Yellow oil. ${ }^{1} \mathrm{H}$ NMR (400 $\left.\mathrm{MHz}, \mathrm{CDCl}_{3}\right): \delta=9.49(\mathrm{~s}, 1 \mathrm{H}), 7.32(\mathrm{~d}, J=2.3 \mathrm{~Hz}, 1 \mathrm{H}), 7.00(\mathrm{~d}, J=4.1 \mathrm{~Hz}, 1 \mathrm{H}), 4.99(\mathrm{~m}, 1 \mathrm{H})$, $4.85(\mathrm{dd}, J=13.2,1.4 \mathrm{~Hz}, 1 \mathrm{H}), 4.54(\mathrm{dd}, \mathrm{J}=13.2,4.9 \mathrm{~Hz}, 1 \mathrm{H}), 4.04(\mathrm{~s}, 1 \mathrm{H}), 1.17$ (s, 3H), 1.16 (s, $3 \mathrm{H}) ;{ }^{13} \mathrm{C}$ NMR $\left(100 \mathrm{MHz}, \mathrm{CDCl}_{3}\right): \delta=202.90,148.70,129.86,128.67,126.45,123.42,121.25$, 80.04, 65.50, 49.45, 42.62, 20.85, 20.40; IR (KBr): 2920, 2851, 1719, 1553, 1468, 1246, 1184 , $1026,866 \mathrm{~cm}^{-1}$; MS (ESI): $\mathrm{m} / z=316.0$ [M - H] $;$ HRMS (ESI) calcd for $\mathrm{C}_{13} \mathrm{H}_{12} \mathrm{Cl}_{2} \mathrm{NO}_{4}^{-}[\mathrm{M}-\mathrm{H}]^{-}:$ 316.0143, found: 316.0150 .

trans-2-(6,8-Dibromo-3-nitrochroman-4-yl)-2-methylpropanal (3j). Yellow oil. ${ }^{1} \mathrm{H}$ NMR $\left(400 \mathrm{MHz}, \mathrm{CDCl}_{3}\right): \delta=9.48(\mathrm{~s}, 1 \mathrm{H}), 7.62(\mathrm{~d}, J=2.1 \mathrm{~Hz}, 1 \mathrm{H}), 7.18(\mathrm{~d}, J=2.0 \mathrm{~Hz}, 1 \mathrm{H}), 4.98(\mathrm{~m}$, 1H), 4.84 (ddd, $J=13.2,4.3,1.5 \mathrm{~Hz}, 1 \mathrm{H}), 4.54$ (dd, J = 13.2, $5.0 \mathrm{~Hz}, 1 \mathrm{H}$ ), 4.03 (s, 1H), 1.17 (s, $3 \mathrm{H}), 1.16(\mathrm{~s}, 3 \mathrm{H}) ;{ }^{13} \mathrm{C}$ NMR $\left(100 \mathrm{MHz}, \mathrm{CDCl}_{3}\right): \delta=202.88,150.13,135.37,132.26,121.66$, 113.79, 112.45, 80.09, 65.60, 49.46, 42.72, 20.85, 20.35; IR (KBr): 2920, 2851, 1718, 1551, 1464, 1356, 1242, 1170, $722 \mathrm{~cm}^{-1}$; MS (ESI): $\mathrm{m} / \mathrm{z}=403.9[\mathrm{M}-\mathrm{H}]^{-}$; HRMS (ESI) calcd for $\mathrm{C}_{13} \mathrm{H}_{12} \mathrm{Br}_{2} \mathrm{NO}_{4}^{-}[\mathrm{M}-\mathrm{H}]^{-}:$403.9133, found: 403.9135 .

trans-2-(3-Nitrochroman-4-yl)acetaldehyde (4b). Yellow oil. ${ }^{1} \mathrm{H}$ NMR (400 $\left.\mathrm{MHz}, \mathrm{CDCl}_{3}\right)$ : $\delta=9.83(\mathrm{~s}, 1 \mathrm{H}), 7.20-7.14(\mathrm{~m}, 1 \mathrm{H}), 7.12(\mathrm{~d}, J=7.7 \mathrm{~Hz}, 1 \mathrm{H}), 6.97(\mathrm{td}, J=7.6,1.2 \mathrm{~Hz}, 1 \mathrm{H}), 6.86$ $(\mathrm{dd}, J=8.2,1.0 \mathrm{~Hz}, 1 \mathrm{H}), 4.84-4.80(\mathrm{~m}, 1 \mathrm{H}), 4.77(\mathrm{ddd}, J=12.1,4.2,1.5 \mathrm{~Hz}, 1 \mathrm{H}), 4.32(\mathrm{dd}, J=$ $12.1,2.3 \mathrm{~Hz}, 1 \mathrm{H}$ ), $4.28-4.22$ (m, 1H), 3.10 (dd, $J=18.9,4.4 \mathrm{~Hz}, 1 \mathrm{H}), 2.90$ (ddd, $J=18.9,9.0$, $0.7 \mathrm{~Hz}, 1 \mathrm{H}) ;{ }^{13} \mathrm{C}$ NMR $\left(100 \mathrm{MHz}, \mathrm{CDCl}_{3}\right): \delta=198.41,153.01,128.65,128.59,122.27,120.88$, 117.37, 81.31, 63.41, 49.45, 31.24; IR (KBr): 2961, 2851, 1716, 1609, 1585, 1541, 1489, 1459, $1363,1222,1042,756 \mathrm{~cm}^{-1}$; MS (ESI): $\mathrm{m} / z=220.1[\mathrm{M}-\mathrm{H}]^{-}$; HRMS (ESI) calcd for $\mathrm{C}_{11} \mathrm{H}_{10} \mathrm{NO}_{4}{ }^{-}$ $[\mathrm{M}-\mathrm{H}]^{-}: 220.0610$, found: 220.0617.

2-(3-Nitrochroman-4-yl)propanal (4c). Colorless oil. $\mathrm{Dr}=71: 29$, signals corresponding to the major diastereoisomer: ${ }^{1} \mathrm{H}$ NMR $\left(400 \mathrm{MHz}, \mathrm{CDCl}_{3}\right): \delta=9.77(\mathrm{~d}, J=1.0 \mathrm{~Hz}, 1 \mathrm{H}), 7.23-6.85(\mathrm{~m}$, $4 \mathrm{H}$ ), 4.90 (dd, $J=8.1,4.6 \mathrm{~Hz}, 1 \mathrm{H}), 4.72(\mathrm{ddd}, J=12.4,4.8,1.3 \mathrm{~Hz}, 1 \mathrm{H}), 4.42(\mathrm{dd}, \mathrm{J}=12.4,3.5$ $\mathrm{Hz}, 1 \mathrm{H}), 4.13(\mathrm{t}, J=5.5 \mathrm{~Hz}, 1 \mathrm{H}), 2.85-2.75(\mathrm{~m}, 1 \mathrm{H}), 1.18(\mathrm{~d}, J=7.3 \mathrm{~Hz}, 3 \mathrm{H}) ;{ }^{13} \mathrm{C}$ NMR $(100$ $\left.\mathrm{MHz}, \mathrm{CDCl}_{3}\right): \delta=202.43,154.06,129.60,128.78,121.81,118.45,117.47,80.80,64.01,49.96$, $37.88,11.25$; signals corresponding to the minor diastereoisomer: ${ }^{1} \mathrm{H}$ NMR $\left(400 \mathrm{MHz}, \mathrm{CDCl}_{3}\right)$ : $\delta=9.79(\mathrm{~d}, J=0.8 \mathrm{~Hz}, 1 \mathrm{H}), 7.23-6.85(\mathrm{~m}, 4 \mathrm{H}), 4.77$ (dd, $J=8.1,4.6 \mathrm{~Hz}, 1 \mathrm{H}), 4.73$ (ddd, $J=$ $12.4,4.8,1.3 \mathrm{~Hz}, 1 \mathrm{H}), 4.55(\mathrm{dd}, \mathrm{J}=12.4,3.5 \mathrm{~Hz}, 1 \mathrm{H}), 4.28(\mathrm{t}, J=5.5 \mathrm{~Hz}, 1 \mathrm{H}), 3.11-2.97(\mathrm{~m}$, 
$1 \mathrm{H}), 1.16(\mathrm{~d}, J=7.4 \mathrm{~Hz}, 3 \mathrm{H}) ;{ }^{13} \mathrm{C} \mathrm{NMR}\left(100 \mathrm{MHz}, \mathrm{CDCl}_{3}\right): \delta=201.86,153.50,129.02,128.38$, 122.38, 119.46, 117.62, 80.39, 64.91, 50.57, 36.17, 10.12; IR (KBr): 2923, 2852, 1718, 1609, $1585,1551,1490,1458,1361,1225,1121,1043,858,757 \mathrm{~cm}^{-1}$; MS (ESI): $\mathrm{m} / z=234.1[\mathrm{M}-\mathrm{H}]^{-}$; HRMS (ESI) calcd for $\mathrm{C}_{12} \mathrm{H}_{12} \mathrm{NO}_{4}^{-}[\mathrm{M}-\mathrm{H}]^{-}: 234.0766$, found: 234.0769 .

2-(3-Nitrochroman-4-yl)butanal (4d). Colorless oil. $\mathrm{Dr}=60: 40$, signals corresponding to the major diastereoisomer: ${ }^{1} \mathrm{H}$ NMR $\left(400 \mathrm{MHz}, \mathrm{CDCl}_{3}\right)$ : $\delta=9.73(\mathrm{~d}, J=2.1 \mathrm{~Hz}, 1 \mathrm{H}), 7.24-6.82(\mathrm{~m}$, $4 \mathrm{H}), 4.86(\mathrm{q}, J=3.7 \mathrm{~Hz}, 1 \mathrm{H}), 4.74(\mathrm{ddd}, J=10.4,4.1,1.6 \mathrm{~Hz}, 1 \mathrm{H}), 4.36(\mathrm{dd}, \mathrm{J}=12.6,3.5 \mathrm{~Hz}$, $1 \mathrm{H}), 4.06(\mathrm{dd}, J=7.3,3.4 \mathrm{~Hz}, 1 \mathrm{H}), 2.66$ (dddd, $J=9.0,7.3,4.9,2.1 \mathrm{~Hz}, 1 \mathrm{H}), 1.64-1.49$ (m, 2H), $1.00(\mathrm{t}, J=7.5 \mathrm{~Hz}, 3 \mathrm{H}) ;{ }^{13} \mathrm{C} \mathrm{NMR}\left(100 \mathrm{MHz}, \mathrm{CDCl}_{3}\right): \delta=203.28,153.41,128.09,128.63,121.77$, $118.63,117.52,80.54,63.77,56.91,37.06,19.97,11.92$; signals corresponding to the minor diastereoisomer: ${ }^{1} \mathrm{H}$ NMR (400 MHz, $\left.\mathrm{CDCl}_{3}\right): \delta 9.75(\mathrm{~d}, J=2.3 \mathrm{~Hz}, 1 \mathrm{H}), 7.24-6.82(\mathrm{~m}, 4 \mathrm{H})$, 4.80 (q, $J=3.4 \mathrm{~Hz}, 1 \mathrm{H}), 4.77$ (ddd, $J=12.4,4.8,1.3 \mathrm{~Hz}, 1 \mathrm{H}), 4.32(\mathrm{dd}, \mathrm{J}=12.3,3.2 \mathrm{~Hz}, 1 \mathrm{H})$, 4.20 (dd, $J=7.3,3.4 \mathrm{~Hz}, 1 \mathrm{H}), 2.76$ (dddd, $J=9.0,7.3,4.9,2.1 \mathrm{~Hz}, 1 \mathrm{H}), 1.91-1.74(\mathrm{~m}, 2 \mathrm{H}), 0.97$ $(\mathrm{t}, J=7.4 \mathrm{~Hz}, 3 \mathrm{H}) ;{ }^{13} \mathrm{C} \mathrm{NMR}\left(100 \mathrm{MHz}, \mathrm{CDCl}_{3}\right): \delta 202.25,153.80,129.59,128.90,122.25$, 119.17, 117.59, 80.54, 64.45, 57.50, 36.53, 19.52, 12.32; IR (KBr): 2965, 1717, 1609, 1585, 1556, 1491, 1462, 1388, 1361, 1232, 1122, 1044, 857, $758 \mathrm{~cm}^{-1}$; MS (ESI): $\mathrm{m} / z=248.1$ [M - H] HRMS (ESI) calcd for $\mathrm{C}_{13} \mathrm{H}_{14} \mathrm{NO}_{4}^{-}[\mathrm{M}-\mathrm{H}]^{-}: 248.0923$, found: 248.0929.

trans-1-(3-Nitrochroman-4-yl)cyclopentanecarbaldehyde (4e). White solid, Mp 95.1-96.7 ${ }^{\circ} \mathrm{C}$. ${ }^{1} \mathrm{H}$ NMR $\left(400 \mathrm{MHz}, \mathrm{CDCl}_{3}\right): \delta=9.39(\mathrm{~s}, 1 \mathrm{H}), 7.25-7.16(\mathrm{~m}, 2 \mathrm{H}), 7.06-6.92(\mathrm{~m}, 1 \mathrm{H}), 6.88(\mathrm{~d}$, $J=8.5,1 \mathrm{H}), 4.85(\mathrm{dt}, J=4.2,2.7,1 \mathrm{H}), 4.81-4.71(\mathrm{~m}, 1 \mathrm{H}), 4.32(\mathrm{dd}, J=13.3,4.3,1 \mathrm{H}), 3.96$ $(\mathrm{s}, 1 \mathrm{H}), 2.25(\mathrm{t}, J=7.9,1 \mathrm{H}), 2.11(\mathrm{dd}, J=12.7,7.4,1 \mathrm{H}), 1.86-1.34(\mathrm{~m}, 6 \mathrm{H}) ;{ }^{13} \mathrm{C} \mathrm{NMR}(100$ $\left.\mathrm{MHz}, \mathrm{CDCl}_{3}\right): \delta=203.75,153.75,130.24,129.55,121.70,117.67,117.37,81.26,63.83,61.58$, 43.79, 32.45, 30.86, 24.28, 23.76; IR (KBr): 2923, 2853, 1718, 1608, 1584, 1553, 1489, 1457 , 1392, 1361, 1228, 1122, 1044, $756 \mathrm{~cm}^{-1}$; MS (ESI): $\mathrm{m} / z=274.1$ [M - H] ; HRMS (ESI) calcd for $\mathrm{C}_{15} \mathrm{H}_{16} \mathrm{NO}_{4}^{-}[\mathrm{M}-\mathrm{H}]^{-}: 274.1079$, found: 274.1081 .

2-(3-Nitrochroman-4-yl)cyclopentanone (4f). Brown oil. $\mathrm{Dr}=84: 16$, signals corresponding to the major diastereoisomer: ${ }^{1} \mathrm{H} \mathrm{NMR}\left(400 \mathrm{MHz}, \mathrm{CDCl}_{3}\right): \delta=7.24-6.81(\mathrm{~m}, 3 \mathrm{H}), 6.96(\mathrm{dd}, J=7.8$, $1.6 \mathrm{~Hz}, 1 \mathrm{H}), 5.34$ (q, $J=3.7 \mathrm{~Hz}, 1 \mathrm{H}), 4.89$ (ddd, $J=12.8,3.3,1.8 \mathrm{~Hz}, 1 \mathrm{H}), 4.43$ (dd, J = 12.8, $3.1 \mathrm{~Hz}, 1 \mathrm{H}), 4.13-4.04(\mathrm{~m}, 1 \mathrm{H}), 2.48-2.37(\mathrm{~m}, 1 \mathrm{H}), 2.34-2.25(\mathrm{~m}, 1 \mathrm{H}), 2.24-2.12(\mathrm{~m}, 1 \mathrm{H})$, $2.09-1.98(\mathrm{~m}, 2 \mathrm{H}), 1.84-1.62(\mathrm{~m}, 2 \mathrm{H}) ;{ }^{13} \mathrm{C} \mathrm{NMR}\left(100 \mathrm{MHz}, \mathrm{CDCl}_{3}\right): \delta=218.30,153.27$, 129.62, 129.10, 121.73, 119.41, 117.44, 81.07, 63.62, 53.15, 38.87, 37.73, 27.97, 20.51; signals corresponding to the minor diastereoisomer: ${ }^{1} \mathrm{H} \mathrm{NMR}\left(400 \mathrm{MHz}, \mathrm{CDCl}_{3}\right): \delta=7.24-6.81(\mathrm{~m}, 3 \mathrm{H})$, $7.00(\mathrm{dd}, J=7.5,1.2 \mathrm{~Hz}, 1 \mathrm{H}) .4 .72-4.67(\mathrm{~m}, 1 \mathrm{H}), 4.61$ (ddd, $J=11.7,5.3,1.1 \mathrm{~Hz}, 1 \mathrm{H}), 4.32$ $(\mathrm{dd}, \mathrm{J}=11.8,3.0 \mathrm{~Hz}, 1 \mathrm{H}), 4.27(\mathrm{t}, J=4.5 \mathrm{~Hz}, 1 \mathrm{H}), 2.82-2.74(\mathrm{~m}, 1 \mathrm{H}), 2.34-2.25(\mathrm{~m}, 1 \mathrm{H})$, $2.24-2.12(\mathrm{~m}, 1 \mathrm{H}), 2.09-1.98(\mathrm{~m}, 2 \mathrm{H}), 1.84-1.62(\mathrm{~m}, 2 \mathrm{H}) ;{ }^{13} \mathrm{C} \mathrm{NMR}\left(100 \mathrm{MHz}, \mathrm{CDCl}_{3}\right)$ : $\delta=216.72,154.03,128.56,128.17,122.50,121.14,117.63,81.07,65.68,54.76,38.03,35.48$, 26.08, 20.59; IR (KBr): 2923, 2853, 1734, 1583, 1544, 1460, 1362, 1223, $756 \mathrm{~cm}^{-1}$; MS (ESI): $m / z=260.1[\mathrm{M}-\mathrm{H}]^{-}$; HRMS (ESI) calcd for $\mathrm{C}_{14} \mathrm{H}_{14} \mathrm{NO}_{4}^{-}[\mathrm{M}-\mathrm{H}]^{-}: 260.0923$, found: 260.0927.

2-(3-Nitrochroman-4-yl)cyclohexanone (4g). White solid, Mp 92.6-93.8 ${ }^{\circ} \mathrm{C}$. Dr = 57:43, signals corresponding to the major diastereoisomer: ${ }^{1} \mathrm{H}$ NMR (400 $\left.\mathrm{MHz}, \mathrm{CDCl}_{3}\right): \delta=7.21-6.83$ 
(m, 4H), $4.99(\mathrm{q}, J=3.6 \mathrm{~Hz}, 1 \mathrm{H}), 4.80(\mathrm{ddd}, J=12.6,3.6,1.5 \mathrm{~Hz}, 1 \mathrm{H}), 4.41(\mathrm{dd}, \mathrm{J}=12.6,3.5 \mathrm{~Hz}$, $1 \mathrm{H}), 4.10(\mathrm{dd}, J=8.7,3.7 \mathrm{~Hz}, 1 \mathrm{H}), 3.04-2.47(\mathrm{~m}, 2 \mathrm{H}), 2.38(\mathrm{dtd}, J=21.7,13.2,5.8 \mathrm{~Hz}, 1 \mathrm{H})$, $2.13(\mathrm{tdd}, J=12.5,5.9,2.9 \mathrm{~Hz}, 1 \mathrm{H}), 2.03-1.87(\mathrm{~m}, 2 \mathrm{H}), 1.81-1.31(\mathrm{~m}, 3 \mathrm{H}) ;{ }^{13} \mathrm{C}$ NMR $(100$ $\left.\mathrm{MHz}, \mathrm{CDCl}_{3}\right): \delta=211.14,154.79,130.83,128.89,122.61,121.35,117.34,81.30,63.79,54.18$, $42.89,37.26,32.27,28.15,25.33$; signals corresponding to the minor diastereoisomer: ${ }^{1} \mathrm{H} \mathrm{NMR}$ $\left(400 \mathrm{MHz}, \mathrm{CDCl}_{3}\right): \delta=7.21-6.83(\mathrm{~m}, 4 \mathrm{H}), 4.89(\mathrm{dd}, J=5.33 .3 \mathrm{~Hz}, 1 \mathrm{H}), 4.70$ (ddd, $J=12.3$, $3.6,1.4 \mathrm{~Hz}, 1 \mathrm{H}), 4.35(\mathrm{~s}, 1 \mathrm{H}), 4.14(\mathrm{dd}, \mathrm{J}=12.4,3.1 \mathrm{~Hz}, 1 \mathrm{H}), 3.04-2.47(\mathrm{~m}, 2 \mathrm{H}), 2.38(\mathrm{dtd}, J=$ 21.7, 13.2, $5.8 \mathrm{~Hz}, 1 \mathrm{H}), 2.13$ (tdd, $J=12.5,5.9,2.9 \mathrm{~Hz}, 1 \mathrm{H}), 2.03-1.87$ (m, 2H), $1.81-1.31$ (m, $3 \mathrm{H}) ;{ }^{13} \mathrm{C}$ NMR $\left(100 \mathrm{MHz}, \mathrm{CDCl}_{3}\right): \delta=210.59,153.44,128.10,128.33,121.03,119.28,117.64$, 81.91, 66.42, 55.45, 42.30, 34.38, 29.49, 27.57, 25.72; IR (KBr): 2925, 2855, 1703, 1584, 1549, 1489, 1451, 1358, 1309, 1224, 1124, $757 \mathrm{~cm}^{-1}$; MS (ESI): $\mathrm{m} / z=274.1$ [M - H] ; HRMS (ESI) calcd for $\mathrm{C}_{15} \mathrm{H}_{16} \mathrm{NO}_{4}^{-}[\mathrm{M}-\mathrm{H}]^{-}: 274.1079$, found: 274.1084 .

trans-1-(3-Nitrochroman-4-yl)propan-2-one (4h). Colorless oil. ${ }^{1} \mathrm{H}$ NMR (400 $\left.\mathrm{MHz}, \mathrm{CDCl}_{3}\right)$ : $\delta=7.19-7.12(\mathrm{~m}, 1 \mathrm{H}), 7.10(\mathrm{~d}, J=7.7 \mathrm{~Hz}, 1 \mathrm{H}), 6.96(\mathrm{td}, J=7.6,1.2 \mathrm{~Hz}, 1 \mathrm{H}), 6.85(\mathrm{dd}, J=8.2$, $1.1 \mathrm{~Hz}, 1 \mathrm{H}), 4.85(\mathrm{dd}, J=6.1,3.5 \mathrm{~Hz}, 1 \mathrm{H}), 4.77$ (ddd, $J=12.3,3.9,1.6 \mathrm{~Hz}, 1 \mathrm{H}), 4.29(\mathrm{dd}, J=$ $12.3,2.4 \mathrm{~Hz}, 1 \mathrm{H}), 4.20-4.12(\mathrm{~m}, 1 \mathrm{H}), 3.02(\mathrm{dd}, J=18.5,4.2 \mathrm{~Hz}, 1 \mathrm{H}), 2.81(\mathrm{dd}, J=18.5,9.3 \mathrm{~Hz}$, $1 \mathrm{H}), 2.21(\mathrm{~s}, 3 \mathrm{H}) ;{ }^{13} \mathrm{C} \mathrm{NMR}\left(100 \mathrm{MHz}, \mathrm{CDCl}_{3}\right): \delta=205.13,153.00,128.63,128.44,122.14$, 121.47, 117.26, 81.38, 63.37, 49.18, 32.29, 30.27; IR (KBr): 2921, 2851, 1716, 1609, 1585, 1542, 1489, 1459, 1361, 1222, 1041, $756 \mathrm{~cm}^{-1}$; MS (ESI): $\mathrm{m} / \mathrm{z}=234.1$ [M - H] ; HRMS (ESI) calcd for $\mathrm{C}_{12} \mathrm{H}_{12} \mathrm{NO}_{4}^{-}[\mathrm{M}-\mathrm{H}]^{-*}: 234.0766$, found: 234.0769.

trans-2-(3-Nitrochroman-4-yl)-1-phenylethanone (4i). White solid, Mp 86.4-88.2 ${ }^{\circ} \mathrm{C} .{ }^{1} \mathrm{H}$ NMR $\left(400 \mathrm{MHz}, \mathrm{CDCl}_{3}\right): \delta=7.96(\mathrm{dd}, J=8.3,1.2 \mathrm{~Hz}, 2 \mathrm{H}), 7.68-7.57(\mathrm{~m}, 1 \mathrm{H}), 7.49$ (dd, $J=10.7,4.7$ $\mathrm{Hz}, 2 \mathrm{H}), 7.22-7.13(\mathrm{~m}, 2 \mathrm{H}), 7.01-6.93(\mathrm{~m}, 1 \mathrm{H}), 6.88(\mathrm{dd}, J=8.6,1.0 \mathrm{~Hz}, 1 \mathrm{H}), 4.96(\mathrm{dd}, J=$ 6.0, $2.7 \mathrm{~Hz}, 1 \mathrm{H}), 4.84(\mathrm{ddd}, J=12.4,3.6,1.6 \mathrm{~Hz}, 1 \mathrm{H}), 4.39(\mathrm{~d}, J=10.3 \mathrm{~Hz}, 1 \mathrm{H}), 4.35(\mathrm{dd}, J=$ 12.4, $2.4 \mathrm{~Hz}, 1 \mathrm{H}), 3.59(\mathrm{dd}, J=18.4,3.8 \mathrm{~Hz}, 1 \mathrm{H}), 3.33(\mathrm{dd}, J=18.4,10.1 \mathrm{~Hz}, 1 \mathrm{H}) ;{ }^{13} \mathrm{C} \mathrm{NMR}$ $\left(100 \mathrm{MHz}, \mathrm{CDCl}_{3}\right): \delta=196.69,153.32,136.25,134.11,129.09,129.06,128.65,128.27,122.37$, 121.92, 117.49, 81.66, 63.52, 44.90, 32.60; IR (KBr): 2921, 2851, 1678, 1583, 1549, 1489, 1448, 1355, 1222, 1103, 754, $689 \mathrm{~cm}^{-1}$; MS (ESI): $\mathrm{m} / z=296.1[\mathrm{M}-\mathrm{H}]^{-}$; HRMS (ESI) calcd for $\mathrm{C}_{17} \mathrm{H}_{14} \mathrm{NO}_{4}^{-}[\mathrm{M}-\mathrm{H}]^{-}: 296.0923$, found: 296.0913 .

1-Methoxy-1-(3-nitrochroman-4-yl)propan-2-one (4j). Brown oil. Dr $=55: 45$, signals corresponding to the major diastereoisomer: ${ }^{1} \mathrm{H} \mathrm{NMR}\left(400 \mathrm{MHz}, \mathrm{CDCl}_{3}\right): \delta=7.23-6.84(\mathrm{~m}, 4 \mathrm{H})$, $4.92(\mathrm{dd}, J=6.3,3.7 \mathrm{~Hz}, 1 \mathrm{H}), 4.77(\mathrm{ddd}, J=11.7,3.3,1.6 \mathrm{~Hz}, 1 \mathrm{H}), 4.34(\mathrm{dd}, \mathrm{J}=12.5,3.0 \mathrm{~Hz}$, $1 \mathrm{H}), 4.16-4.13(\mathrm{~m}, 1 \mathrm{H}), 3.91(\mathrm{~d}, J=6.1 \mathrm{~Hz}, 1 \mathrm{H}), 3.38(\mathrm{~s}, 3 \mathrm{H}) .2 .37(\mathrm{~s}, 3 \mathrm{H}) ;{ }^{13} \mathrm{C} \mathrm{NMR}(100$ $\left.\mathrm{MHz}, \mathrm{CDCl}_{3}\right): \delta=209.51,153.56,129.10,128.87,121.67,117.55,117.47,88.20,79.52,64.11$, 59.76, 38.97, 27.98; signals corresponding to the minor diastereoisomer: ${ }^{1} \mathrm{H}$ NMR (400 MHz, $\left.\mathrm{CDCl}_{3}\right): \delta=7.23-6.84(\mathrm{~m}, 4 \mathrm{H}), 4.86(\mathrm{dd}, J=5.5,2.8 \mathrm{~Hz}, 1 \mathrm{H}), 4.80(\mathrm{ddd}, J=11.3,3.4,1.8 \mathrm{~Hz}$, $1 \mathrm{H}), 4.46(\mathrm{dd}, \mathrm{J}=12.2,2.7 \mathrm{~Hz}, 1 \mathrm{H}), 4.16-4.13(\mathrm{~m}, 1 \mathrm{H}), 4.07(\mathrm{~d}, J=4.1 \mathrm{~Hz}, 1 \mathrm{H}), 3.27(\mathrm{~s}, 3 \mathrm{H})$. 2.29 (s, 3H); ${ }^{13} \mathrm{C}$ NMR (100 MHz, $\left.\mathrm{CDCl}_{3}\right): \delta=207.86,154.05,129.73,128.49,121.96,118.21$, 116.73, 89.07, 79.27, 66.91, 59.16, 37.99, 27.47; IR (KBr): 2921, 2851, 1717, 1542, 1508, 1458, 
1362, 1100, $753 \mathrm{~cm}^{-1}$; MS (ESI): $\mathrm{m} / z=264.1$ [M - H] ; HRMS (ESI) calcd for $\mathrm{C}_{13} \mathrm{H}_{14} \mathrm{NO}_{5}^{-}[\mathrm{M}-$ $\mathrm{H}]^{-}: 264.0872$, found: 264.0877 .

1-Hydroxy-1-(3-nitrochroman-4-yl)propan-2-one (4k). Pale yellow oil. $\mathrm{Dr}=60: 40$, signals corresponding to the major diastereoisomer: ${ }^{1} \mathrm{H}$ NMR $\left(400 \mathrm{MHz}, \mathrm{CDCl}_{3}\right): \delta=7.25-6.87(\mathrm{~m}$, 4H), $4.82(\mathrm{ddd}, J=12.3,2.9,1.6 \mathrm{~Hz}, 1 \mathrm{H}), 4.67(\mathrm{t}, J=2.9 \mathrm{~Hz}, 1 \mathrm{H}), 4.57(\mathrm{dd}, J=5.1,2.8 \mathrm{~Hz}$, 1H). $4.47(\mathrm{dd}, J=12.3,2.8 \mathrm{~Hz}, 1 \mathrm{H}), 4.16(\mathrm{~s}, 1 \mathrm{H}), 3.50$ (s, 1H). $2.37(\mathrm{~s}, 3 \mathrm{H}) ;{ }^{13} \mathrm{C}$ NMR $(100$ $\left.\mathrm{MHz}, \mathrm{CDCl}_{3}\right): \delta=207.98,154.28,128.99,128.27,122.25,117.77,117.67,79.81,79.20,64.99$, 39.07, 26.43; signals corresponding to the minor diastereoisomer: ${ }^{1} \mathrm{H}$ NMR $\left(400 \mathrm{MHz}, \mathrm{CDCl}_{3}\right)$ : $\delta=7.25-6.87(\mathrm{~m}, 4 \mathrm{H}), 4.93(\mathrm{dd}, J=6.4,3.0 \mathrm{~Hz}, 1 \mathrm{H}), 4.77(\mathrm{ddd}, J=12.3,3.7,1.6 \mathrm{~Hz}, 1 \mathrm{H}), 4.52$ $(\mathrm{dd}, \mathrm{J}=12.4,2.8 \mathrm{~Hz}, 1 \mathrm{H}), 4.51(\mathrm{t}, J=2.9 \mathrm{~Hz}, 1 \mathrm{H}), 4.29(\mathrm{~s}, 1 \mathrm{H}), 3.51(\mathrm{~s}, 1 \mathrm{H}), 2.26(\mathrm{~s}, 3 \mathrm{H}) ;{ }^{13} \mathrm{C}$ NMR $\left(100 \mathrm{MHz}, \mathrm{CDCl}_{3}\right): \delta=207.38,154.17,129.34,128.46,121.82,117.86,115.66,80.98$, 79.28, 64.35, 40.05, 26.76; IR (KBr): 3448, 2925, 2853, 1716, 1609, 1586, 1552, 1491, 1461, $1225,1103,1041,758 \mathrm{~cm}^{-1}$; MS (ESI): $\mathrm{m} / z=250.0$ [M - H] ; HRMS (ESI) calcd for $\mathrm{C}_{12} \mathrm{H}_{12} \mathrm{NO}_{5}{ }^{-}$ $[\mathrm{M}-\mathrm{H}]^{-}: 250.0715$, found: 250.0720 .

\section{Reduction of trans-2-(3-nitrochroman-4-yl)-2-methylpropanal (3a) with zinc/AcOH ${ }^{13}$}

To a solution of the 3a $(62 \mathrm{mg}, 0.25 \mathrm{mmol})$ in $\mathrm{MeOH} / \mathrm{AcOH}(V / V=1 / 1,5 \mathrm{~mL})$ at $0{ }^{\circ} \mathrm{C}$ was added zinc powder $(400 \mathrm{mg}, 6.25 \mathrm{mmol})$ in small portions over a period of $5 \mathrm{~min}$. The reaction mixture was stirred for $2 \mathrm{~h}$ at room temperature and was then filtered. The filtrate was adjusted to $\mathrm{pH} 12$ with aqueous $\mathrm{NaOH}(4 \mathrm{M})$. The aqueous layer was extracted with EtOAc $(20 \mathrm{~mL} \times 3)$. The combined organic layer was dried over anhydrous $\mathrm{Na}_{2} \mathrm{SO}_{4}$. After the solvent was evaporated under vacuum, the residue was purified by flash column chromatography over silica gel $\left(\mathrm{CH}_{2} \mathrm{Cl}_{2} / \mathrm{MeOH}=20 / 1\right)$ to provide cis-1,1-dimethyl-1,2,3,3a,4,9b-hexahydrochromeno[3,4b]pyrrole (5) as a colorless oil. ${ }^{1} \mathrm{H}$ NMR $(400 \mathrm{MHz}, \mathrm{MeOD}): \delta=7.24(\mathrm{~d}, J=7.7 \mathrm{~Hz}, 1 \mathrm{H}), 7.08$ $(\mathrm{dd}, J=7.5,4.6 \mathrm{~Hz}, 1 \mathrm{H}), 6.85(\mathrm{dd}, J=7.5,1.1 \mathrm{~Hz}, 1 \mathrm{H}), 6.75(\mathrm{~d}, J=8.2 \mathrm{~Hz}, 1 \mathrm{H}), 4.48(\mathrm{dd}, J=$ 9.4, $4.1 \mathrm{~Hz}, 1 \mathrm{H}), 4.10(\mathrm{dd}, J=10.8,9.5 \mathrm{~Hz}, 1 \mathrm{H}), 3.50(\mathrm{td}, J=11.6,3.7 \mathrm{~Hz}, 1 \mathrm{H}), 3.30(\mathrm{dd}, J=1.6$, $3.2 \mathrm{~Hz}, 1 \mathrm{H}), 3.10(\mathrm{~d}, J=11.2 \mathrm{~Hz}, 1 \mathrm{H}), 3.02(\mathrm{~d}, J=11.2 \mathrm{~Hz}, 1 \mathrm{H}), 2.71(\mathrm{~d}, J=11.6 \mathrm{~Hz}, 1 \mathrm{H}), 1.47$ (s, 3H), 1.07 (s, 3H); ${ }^{13} \mathrm{C}$ NMR (100 MHz, MeOD): $\delta=155.69,128.91,127.27,123.35,121.24$, 117.49, 70.96, 62.52, 58.12, 51.87, 40.68, 28.78, 22.52; IR (KBr): 3444, 2961, 2929, 2878, 1609 , 1486, 1452, 1230, 1160, 1074, 953, 860, $754 \mathrm{~cm}^{-1}$; MS (ESI): $\mathrm{m} / \mathrm{z}=204.1[\mathrm{M}+\mathrm{H}]^{-} ; \mathrm{HRMS}$ (ESI) calcd for $\mathrm{C}_{13} \mathrm{H}_{18} \mathrm{NO}^{+}[\mathrm{M}+\mathrm{H}]^{+}:$204.1388, found: 204.1378 .

\section{Acknowledgements}

We thank National Natural Science Foundation of China (No. 20772160, 20972195), Ministry of Science and Technology of China (No. 2009ZX09501-017) for the financial support of this study. 


\section{References}

1. (a) Mellin, C.; Vallgaarda, J.; Nelson, D. L.; Bjoerk, L.; Yu, H.; Anden, N. E.; Csoeregh, I.; Arvidsson, L. E.; Hacksell, U. J. Med. Chem. 1991, 34, 497. (b) Podona, T.; GuardiolaLemaitre, B.; Caignard, D. H.; Adam, G.; Pfeiffer, B.; Renard, P.; Guillaumet, G. J. Med. Chem. 1994, 37, 1779. (c) Comoy, C.; Marot, C.; Podona, T.; Baudin, M. L.; Morin-Allory, L.; Guillaumet, G.; Pfeiffer, B.; Caignard, D. H.; Renard, P.; Rettori, M. C.; Adam, G.; Guardiola-Lemaitre, B. J. Med. Chem. 1996, 39, 4285. (d) Dubuffet, T.; Newman-Tancredi, A.; Cussac, D.; Audinot, V.; Loutz, A.; Millan, M. J.; Lavielle, G. Bioorg. Med. Chem. Lett. 1999, 9, 2059. (e) Cueva, J. P.; Giorgioni, G.; Grubbs, R. A.; Chemel, B. R.; Watts, V. J.; Nichols, D. E. J. Med. Chem. 2006, 49, 6848. (f) Furuta, T.; Hirooka, Y.; Abe, A.; Sugata, Y.; Ueda, M.; Murakami, K.; Suzuki, T.; Tanaka, K.; Kan, T. Bioorg. Med. Chem. Lett. 2007, 17, 3095. (g) Kolokythas, G.; Kostakis, I. K.; Pouli, N.; Marakos, P.; Kousidou, O. C.; Tzanakakis, G. N.; Karamanos, N. K. Eur. J. Med. Chem. 2007, 42, 307.

2. For a recent review of chroman derivatives with biological activity, see: Wang, G. X.; Wang, N. X.; Shi, T.; Yu, J. L.; Tang, X. L. Progress in Chemistry 2008, 20, 518.

3. Kwak, J. H.; Kang, H. E.; Jung, J. K.; Kim, H.; Cho, J.; Lee, H. Arch. Pharm. Res. 2006, 29, 934.

4. Kanbe, Y.; Kim, M. H.; Nishimoto, M.; Ohtake, Y.; Kato, N.; Tsunenari, T.; Taniguchi, K.; Ohizumi, I.; Kaiho, S.; Morikawa, K.; Jo, J. C.; Lim, H. S.; Kim, H. Y. Bioorg. Med. Chem. 2006, 14, 4803.

5. Emami, S.; Kebriaeezadeh, A.; Zamani, M. J.; Shafiee, A. Bioorg. Med. Chem. Lett. 2006, 16, 1803.

6. Koufaki, M.; Kiziridi, C.; Alexi, X.; Alexis, M. N. Bioorg. Med. Chem. 2009, 17, 6432.

7. (a) Wise, L. D.; Dewald, H. A.; Hawkins, E. S.; Reynolds, D. M.; Heffner, T. G.; Meltzer, L. T.; Pugsley, T. A. J. Med. Chem. 1988, 31, 688. (b)Yao, C. F.; Jang, Y. J.; Yan, M. C. Tetrahedron Lett. 2003, 44, 3813. (c) Xu, D. Q.; Wang, Y. F.; Luo, S. P.; Zhang, S.; Zhong, A. G.; Chen, H.; Xu, Z. Y. Adv. Synth. Catal. 2008, 350, 2610. (d) Rao, H. S. P.; Geetha, K. Tetrahedron Lett. 2009, 50, 3836. (e) Wang, Y. F.; Zhang, W.; Luo, S. P.; Li, B. L.; Xia, A. B.; Zhong, A. G.; Xu, D. Q. Chem. Asian J. 2009, 4, 1834.

8. (a) Dauzonne, D.; Royer, R. Synthesis 1984, 348. (b) Neirabeyeh, M. A.; Koussini, R.; Guillaumet, G. Synth. Commun. 1990, 20, 783. (c) Yan, M. C.; Jang, Y. J.; Yao, C. F. Tetrahedron Lett. 2001, 42, 2717. (d) Ballini, R.; Bosica, G.; Fiorini, D.; Palmieri, A. Green Chem. 2005, 7, 825. (e) Koussini, R.; Al-Shihri, A. S. J. Saudi Chem. Soc. 2008, 12, 531.

9. (a) Korotaev, V. Y.; Sosnovskikh, V. Y.; Kutyashev, I. B.; Kodess, M. I. Lett. Org. Chem. 2005, 2, 616. (b) Korotaev, V. Y.; Sosnovskikh, V. Y.; Kutyashev, I. B.; Kodess, M. I. Russ. Chem. Bull. 2006, 55, 2020. (c) Korotaev, V. Y.; Sosnovskikh, V. Y.; Kutyashev, I. B. Russ. Chem. Bull. 2007, 56, 2054. (d) Korotaev, V. Y.; Sosnovskikh, V. Y.; Kutyashev, I. B.; Barkov, A. Y.; Shklyaev, Y. V. Tetrahedron Lett. 2008, 49, 5376. (e) GranadosCovarrubias, E. H.; Maldonado, L. A. J. Org. Chem. 2009, 74, 5097. (f) Habib, P. M.; 
Kavala, V.; Raju, B. R.; Kuo, C. W.; Huang, W. C.; Yao, C. F. Eur. J. Org. Chem. 2009, 4503.

10. (a) Nyerges, M.; Virányi, A.; Marth, G.; Dancsó, A.; Blaskó, G.; Tőke, L. Synlett 2004, 2761. (b) Virányi, A.; Marth, G.; Dancsó, A.; Blaskó, G.; Toke, L.; Nyerges, M. Tetrahedron 2006, 62, 8720. (c) Muruganantham, R.; Mobin, S. M.; Namboothiri, I. N. N. Org. Lett. 2007, 9, 1125. (d) Habib, P. M.; Raju, B. R.; Kavala, V.; Kuo, C. W.; Yao, C. F. Tetrahedron 2009, 65, 5799. (e) Korotaev, V. Y.; Sosnovskikh, V. Y.; Barabanov, M. A.; Yasnova, E. S.; Ezhikova, M. A.; Kodess, M. I.; Slepukhin, P. A. Tetrahedron 2010, 66, 1404.

11. For the reviews of proline and its derivatives as organocatalysts, see: (a) Cheng, C. L.; Hao, E. J.; Li, W.; Xu, G. Q. HuaxueShiji 2007, 29, 147. (b) Kotsuki, H.; Ikishima, H.; Okuyama, A. Heterocycles 2008, 75, 493. (c) Kotsuki, H.; Ikishima, H.; Okuyama, A. Heterocycles 2008, 75, 757.

12. Recently an organocatalytic asymmetric synthesis of 3-nitro-chromans from $o$-hydroxy cinamldehyde and nitroalkenes was reported. See: Zu, L. S.; Zhang, S. L.; Xie, H. X.; Wang, W. Org. Lett. 2009, 11, 1627.

13. Ruiz, N.; Reyes, E.; Vicario, J. L.; Badía, D.; Carrillo, L.; Uria, U. Chem. Eur. J. 2008, 14, 9357. 